L.

Aus dem Institut für Schiffs- und Tropenkrankheiten in Hamburg. (Leiter: Med.-Rath Prof. Dr. Nocht.)

\title{
Qualitative Leukocytenblutbilder mit Einbeziehung der vereinfachten Arneth'schen Methode und ihre plastische Darstellung mit einem Differentialleukocy tometer.
}

\author{
Von \\ Dr. V. Schilling-Torgau, \\ Assistenzarzt, commandirt zum Institut. \\ (Hierza Tafel XXIV.-XXVI und 1 Curve im 'l'ext.)
}

Theorie der Eintheilung der Leukocytenblutbilder.

Die Lehre von der Leukocytose erfreut sich seit Jahrzehnten als ein interessantes und schwieriges Capitel fast aller Zweige der Medicin ciner ausgedehnten Beachtung und Untersuchung. Wir können hier bei der zahlreichen Literatur nur einen ganz kurzen Ueberblick über die Fortschritte auf diesem Gebiete geben. Das Wesentlichste findet sich in den in dieser Hinsicht vorzüglichen Lehrbüchern der Blutkrankheiten von Grawitz (1) und Naegeli (2).

Wie im ganzen Bereiche der Hämatologie lässt sich auf diesem Sondergebiete ein Verlauf feststellen, der einerseits von einer erst oberflächlichen Empirie der Zahlenverhältnisse und der Zellarten zu einem sehr differencirten Studium jeder einzelnen Zelle, andererseits von ganz allgemeinen grossen Erwartungen für die Klinik zu der resignirten Erkenntniss von der principiellen, nur graduell unterschiedenen Gleichheit fast aller Blutbefunde führt.

Die erste Möglichkeit zur gesetzmässigen Erforschung der Jeukocyten wurde durch die Zählverfahren gegeben. Aus vielen Hunderten kasuistischer Mittheilungen und specieller Studien liess sich zwar in grossen Krankheitsgruppen eine leidlich regelmässige Vermehrung bzw. Verminderung der Leukocyten feststellen, aber mit zahllosen individuellen und unerklärlichen Ausnahmen musste der Werth dieser Untersuchungen erheblich sinken.

Line wesentliche Errungenschaft waren die Ehrlich'schen differencirenden Blutfärbungen. Unter Berücksichtigung der charakteristischen Granulationen gelang eine ganz andere Sonderung der Leukocyten, die sich durch histologische Methoden auch auf die Ursprungsstätten, Knochenmark, Lymphdrüsen und Milz ausdehnen liess. Der klinisch bis heute bewährte Dualismus ${ }^{1}$ ) der Leukocyten nach ihrem Ursprunge von cinem

1) Auch von gemässigten Unitariern anerkannt [Pappenheim-Ferrata (33)], embryologisch und vergleichend anatomisch ist $\mathrm{m}$. E. der Unitarismus, d. h. die Abstammung aller Blutelemente von gleichen Stammzellen wohl vertretbar. 
myeloisehen oder lymphatisehen Systeme konnte aufgestellt werden. Damit waren die Grundlagen für Unterscheidung von Lymphocytosen oder speciellen Leukocytosen gegeben. So wurde ein neuer Factor für die Werthung von J.eukocytenveränderung in der möglichen Differentialbeobachtung einzelner Zellklassen geschaffen.

In Folge weiteren Ausbaues der Färbomethoden, insbesondere der Kerndarstellung, wurde diesen beiden wichtigen Untersuchungsmethoden ein dritter wichtiger Factor durch ein genaues morphologisches Studium der Einzel\%elle selbst hinzugefügt. Das vorher nur gelegentlich notirto Auftreten von pathologischen, d. h. in normalem Blute nicht vorkommenden Einzelformen innerhalb einer Zellart ( den Neutrophilen) wurde allgemeiner bemerkt und bei den verschicdensten Krankheiten das Vorhandensein von Myelocyten und Metamyelocyten (Pappenheim) festgestellt. Diese pathologischen Zellformen, die vorher nur bei den eigentlichen Blutkrankheiten, Leukämic usw., als wichtig studirt waren, begannen dic vorher gezogene scharfe Grenze zwischen Leukocytose und Leukämio zu verwischen. In schweren Sepsisfällen, bei Careinose des Markes usw. wurden Blutbilder gefunden, dic den von Leukämien täuschend ähnlich sahen. Andererseits gab es in der Gruppe der l'scudoleukimien oder besser Aleukämien ausgesprochene Krankheiten des hämatopoctischen Apparates ohne charakteristischen Blutbefund. Die Hoffnung, diese Formen durch ein ganz specielles Zellstudium dennoch erkennen zu können, hat sich bisher nicht bewahrheitet, obgleich gewisse Veränderungen in der differentiellen Zusammensetzung (Pinkus'sches Zeichen) nach eingehenderen neueren Untersuchungen [Benjamin (4), Hess (5), Fabian $(6,7)$ u. A.] auch hier Fortschritte erwarten lassen. Gleiches lässt sich von ciner grossen Gruppe von Krankheiten mit weniger charakteristischen Blutveründerungen [z. B. Banti, chronischen Protozoenkrankheiten, Basedow und Basedowoid gegen Struma (Kocher (8), Caro (9) u. A.), Beriberi usw.] sagen. Nur eine sorgfältig ausgefübrte Zählung im Vercin mit differenzirtester Beobachtung der Einzelzellklassen verspricht hier vorläufig Erlolge für die Diagnose.

Die speciell diagnostisch werthvolle Bedeutung der Eosinophilen ist bis heute nur immer befestigt worden [Dunger (10), Stäubli (11) u. A.].

Dennoch erwiesen sich, ebenso wie früher dic Kahlenverhältnisse, auch die Differentialzählungen als nicht ausreichend typisch, um selbst bei Combinationen beider Methoden immer ein sicheres Ergebniss zu gewährleisten. Der von Türck (12) am Schlusse seiner Untersuchungen formulirte Satz, dass alle Blutbilder nur Symptom sind, mithin wie alle klinischen Symptome durch die verschicdensten Ursachen in gleicher Weise ausgelöst wordon können, musste sich mit weiterer Ausdehnung der Kenntnisse nur mehr festigen.

Die Anwendung der histologischen Methoden und der experimentellen Thieruntersuchung zeigte mit immer grösserer Deutlichkoit, dass charakteristische Bofunde im Blute gerade auch bei den einfachen Leukocytosen mit annähernd parallelen Befunden im Knochenmark oder dem lympha- 
tischen System einherzugehen pflegen [vertreten von Naegeli (2) und vielen Anderen, z. B. Pappenheim (Folia hacmatologica), neuerdings Ziegler und Schlecht (14), Bennecke (15) u. A.], dass mithin das periphere Blutbild principiell nur ein Resultat der Umwandlung der hämatopoetischen Organe ist. Allerdings war von Leukümien her bekannt, dass keineswegs ein absoluter Parallelismus vorhanden zu sein braucht.

Die bisherigen Theorien über die Jieukocytose führten die verschiedensten Gründe für die Zunahme oder Abnahme der Leukocyten im peripheren Blute an. Dic Behauptung directer Umwandlung von Lymphocyten zu Leukocyten im peripheren Blute wird heute wohl nur noch von Grawitz $(1,13)$ und soinen Schülern vertretcn. $\Lambda \mathrm{m}$ verbreitesten war die Lehre von der positiven oder negativen Chemotaxis der Leukocyten den verschiedenen schädigenden Ursachen gegenüber; cine Erweiterung dieser Lehre war die Annahme einer in Krankheitsfällen eintretenden veränderten Vortheilung der weissen Blutzellen auf Peripherie und innere Organe (Werigo, Jacob und Goldscheider u. A.) Für die Jeukopenie sehuf Löwit den Begriff der Leukolyse, d. h. einer rapiden Auflösung der kreisenden Leukosyton. In neuerer Zeit begründete Arneth $(16,17)$ die Gesammtlehre von der Leukocytose auf eine Verbrauchs- und Ersatztheorie und glaubte, alle Erscheinungen der Leukocytose durch das Spiel zwisehen Anforderung, Mehrlicferung und Mehrverbrauch der Zellon erklären zu können. Am wenigsten beachtet ist die theoretisch durchaus nothwendige Annahme einer allgemeinen physiologischen Regulirung der Leukocytenzahl und -Zusammensetzung in ähnlicher Weise wie die der Körpertemperatur, der Serumzusammensetzung usw. [Zoja (18], dureh deren krankhafte Störung erst pathologische dauernde Verschicbungen im Zellglcichgewicht möglich wären. Alle diese Jheorien dürften aber nur llilfsbedeutung haben, obgleich jede einzelne hier und da vorwiegende Befunde in besonderen Fïllen für sich anführen kann gegenübes der Lehre von der Leukocytose als einem directen Symptom der Veränderung der hämatopoetischen Organe. Naegeli hat die Jeukopenie des Typhus z. B. in einfacher exporimenteller Weise durch den Nachweis der degenerativen bzw. hemmenden Linwirkung von Typhustoxinen auf das Knochenmark selbst zeigen künnen, viele andere Untersuchungen haben die Vermehrung der Lcukocytenproduction bei septischen Jeukocytosen unzweifelhaft nachgewiesen.

Aus diesen neucren Ansichten resultirt dic ausserordentliche Wichtigkeit, welche eine Erweiterung unserer Kenntnisse von der Morphologie der Einzelzelle besitzen würde, da pathologische Umänderungen, Zeichen cines jugendilicheren Zustandes usw. ganz unabhängrig von Zahl und Zusammensetzung der Leukocyten uns Aufsehlüsse über Veränderungen der hämatopoetischen Organe selbst zu geben vermöchten.

Diesen Weg ist Arneth $(16,17)$ gegangen. Arneth ging von der erwähnten Beobachtung einzolner jugendlicher neutrophiler Leukoeyten und Myelocyten bei Infectionskrankheiten aus und stellte aus der Beobachtung der Kernform der Neutrophilen eine regelmässige durch- 
gehende Veränderung in der gesammten neutrophilen Zellklasse fest, die, unabhängig von Alter und Geschlecht des Patienten, von der Gesammtzahl and Differentialzusammensetzung der Leukocyten eine für gewisse Krankheitsgruppen recht gut übereinstimmende Veränderung der neutrophilen Einzelzellen erkennen liess. A uf Grund folgenden Schemas construirte er eine fortlaufende Reihenfolge nach der Form und Zahl der Kerntheile der neutrophilen Leukocyten.

Arneth'sches neutro-

Kern-

\begin{tabular}{|c|c|c|c|c|c|c|c|c|c|c|c|}
\hline & \multirow{2}{*}{ Zahi } & \multicolumn{3}{|c|}{1} & \multicolumn{3}{|c|}{2} & \multicolumn{4}{|c|}{3} \\
\hline & & M & W & $\mathrm{T}$ & $2 \mathrm{~K}$ & $2 \mathrm{~S}$ & $1 \mathrm{~K} 1 \mathrm{~S}$ & $3 \mathrm{~K}$ & $3 S$ & $2 K 1 \mathrm{~S}$ & $2 S 1 \mathrm{~K}$ \\
\hline i. . & 6000 & - & 0,2 & 5 & 0,27 & 23,5 & 11,7 & 2,27 & 5,6 & 16,7 & 16,4 \\
\hline B. & 9400 & - & - & 5 & 1 & 13 & 16 & 3 & 3 & 25 & 13 \\
\hline C. & 27100 & 1 & 2 & 34 & - & 38 & 15 & 1 & - & 1 & 6 \\
\hline D. & 4200 & - & 5 & 55 & 4 & 21 & 15 & - & - & - & - \\
\hline
\end{tabular}

Nach der Zahl der überhaupt vorhandenen Kerntheile von 1-5 bildete or 5 Hauptklassen, die er in weitere Unterklassen nach der Form der Kerntheilc selbst ( $\mathrm{K}=$ runder Kerntheil, $\mathrm{S}=$ lïnglicher Kerntheil, Schlinge) zerlegte. Nur die erste Klasse, die die "mononucleären" Ncutrophilen gegenüber den polynucleären zusammenfasste, zerlegte er in $M-$ Zellen = Myclocyten, $W$-Zellen = Zellen mit wenig gebuchteten Kornen und $\mathrm{T}$-Zellen $=$ Zellen mit tiefgebuchteten Kernen. Lr behauptete nun, dass jedes Mal die Zelle der früheren Klasse oder Unterabtheilung jünger sei als jede der späteren. Er constatirte, wenn or $100 \mathrm{im}$ mikroskopischen Präparat beobachtete neutrophile Leukocyten in dieses Schema eingetragen hatte, bei vielen Krankheitsfällen eine Verschicbung der Zahl aus den höheren Klassen in die niedrigeren, cine "Verschiebung nach links", die nach seiner Theorie gleichbedeutend mit einer Verjüngung des Blutbildes war. Er sah in dieser Verschiebung den $\Lambda$ usdruck einor vermehrten Bildung jugendlicher Zellen als Resultat einer vermehrten Anforderung an den Verbrauch von Leukocyten. Wenn dieser Verbrauch gedeckt wurde, blieb die Zahl gleich [Normocytose (Schema A)], wenn or übercompensirt wurde, entstand die Hyperleukocytose (B und $C$ ), wenn er nicht gedeckt wurde, dic Hypoleukocytose oder Leukopenie (D). Die Verschiebung in den Kernformen drückte er durch den Vorsatz Iso- oder Aniso- aus (= mit Verschiebung oder ohne Verschiebung). Er fand so, dass os Iso- und AnisoNormocytosen, Iso- und Aniso. Hyperleukocytosen, Isound Aniso-Hypoleukocytoson gab; dabei fand sich, dass die Isoleukocytosen mehr bei physiologischen Veründerungen der Ieukosyten eintraten, während die Anisolenkocytosen die pathologischen Veränderungen der Leukocyten ausdrückten. Mit grosser Deutlichkeit ging aus der sehr ausgedehnten Anwendung dieser Methodik hervor, dass ganz gleiche Zahlenverhältnisse, ja sogar ganz gleich differentiell zusammengesetzte Blutbilder durch das 
Vorhandensein oder Fehlen, sowie durch den Grad einer Verschiebung charakteristisch gesondert wurden. Die histologischen Unterlagen der Arneth'schen Eintheilung nach der Kernform haben einer zahlreichen Kritik nicht standgehalten [s. Sammelkritik SchillingTorgau (44)], das Factum der Verschiebung ist aber nach übereinstimmendem Urtheil unwiderleglich vorhanden. Zur Orientirung füge ich folgende nach Arneth's Buch zusammengestellten Curven philes Blutbild.

teil e.

\begin{tabular}{|c|c|c|c|c|c|c|c|c|c|c|}
\hline \multicolumn{5}{|c|}{4} & \multicolumn{5}{|c|}{5} & \multirow{2}{*}{$\begin{array}{l}\text { Fall der Mono- } \\
\text { graphie } \\
\text { Arneth's }(16) \text { : }\end{array}$} \\
\hline $4 \mathrm{~K}$ & $4 S$ & $3 K 1 S$ & $3 \mathrm{~S} 1 \mathrm{~K}$ & $2 \mathrm{~K} 2 \mathrm{~S}$ & $5 \mathrm{~K}$ & $4 \mathrm{~K}$ IS & $3 \mathrm{~K} 2 \mathrm{~S}$ & $4 \mathrm{~K} 2 \mathrm{~S}$ & $3 \mathrm{~K} 3 \mathrm{~S}$ & \\
\hline 3,8 & 0,07 & 6,4 & 1,6 & 4,73 & 1,0 & 1,4 & 0,4 & 0,07 & 0,07 & Dr. A. \\
\hline 10 & - & 5 & - & 4 & I & 1 & - & 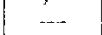 & - & Dr. P.,Verdauung. \\
\hline - & - & - & - & 1 & - & 1 & - & - & - & Fall 53. \\
\hline- & - & - & - & - & - & - & - & - & - & Fall 49 \\
\hline
\end{tabular}

bei (Tabelle S. 692). Aus diesen Curven geht augenscheinlich hervor, dass die Veränderung des Blutbildes immer eine sehr ähnliche ist, dass sie am charakteristischsten in der 1. Klasse gegenüber dem normalen ausgeprägt ist und dass sie sich jm Ganzen wenig durch Auftreten echt jugendlicher Elemente (M- und W-Zellen) ausbildet'1). Die Arneth'schen T-Zellen, dio das Hauptcontigent der Arneth'schen Verschicbungszellen ausmachen, sind meines Erachtens degenerativ schlecht ausgebildete polynucleäre Zcllen, die statt eines gelappten Kernes nur einen stabförmigen pyknotischen Kern zu bilden vermochten. Ich habe diese Ansichten an anderer Stelle unter Würdigung der von vielen Seiten [Zclensky (19), Pappenheim²), Kothe (20) und Sonnenburg (21) u. A.] gemachten Vorschläge zur Verbesserung der Methoden eingehend gewürdigt [Schilling-Torgau (44)] und fasse hier nur kurz die Ergebnisse und den Modus meinor Eintheilung (23) zusammen:

1. Die Zellen von Arneth's 1. Klasse sind nicht alle jugendlich.

2. Unzweifelhaft jugendlich sind:

a) die Myelocyten = M-Zellen,

b) die Metamyelocyten $=\mathrm{W}$-Zellen und der Theil der T-Zellen, die durch einen jugendlich strukturirten Kern, Grösse oder Basophilie des Protoplasmas sich als echte Uebergänge zwischen dem runden Myelocytenkern und dem gelappten Kern des neutrophilen reifen Leukocyten erweisen.

1) Eine Ausnahme bildet F 31 , jedoch ist die Verschiebung in der I. KI. entsprechend der klinischen Bedeutung nicht so extrem. Das Hauptgewicht liegt hier mehr in den lymphocytären Gruppen.

2) Pappenheim, Folia haematologica, IlI. p. 609, V. p. 509, VII. p. 85 und Atlas der menschlichen Blutzellen. 
3. Pathologisch oder degenerativ einkernig sind die Neutrophilen der T-Klasse mit pyknotischem, oft bizarrem Kernstabe, wie sie sich z. B. bei 'l'yphus, bei schwerster Versehiebung, fast ausschliesslich vorfinden. Diesc Zellen habe ich als Stabkernige den Jugendlichen gegenübergestellt, um dadurch eine histologisch und klinisch begründete Trennung der Blutbilder in jugendlich- und pathologischdegenerativ Verschobene zu ermöglichen.

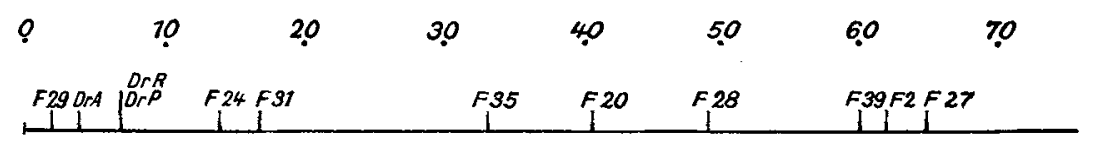

Verschiebung in der L. KI. allein.

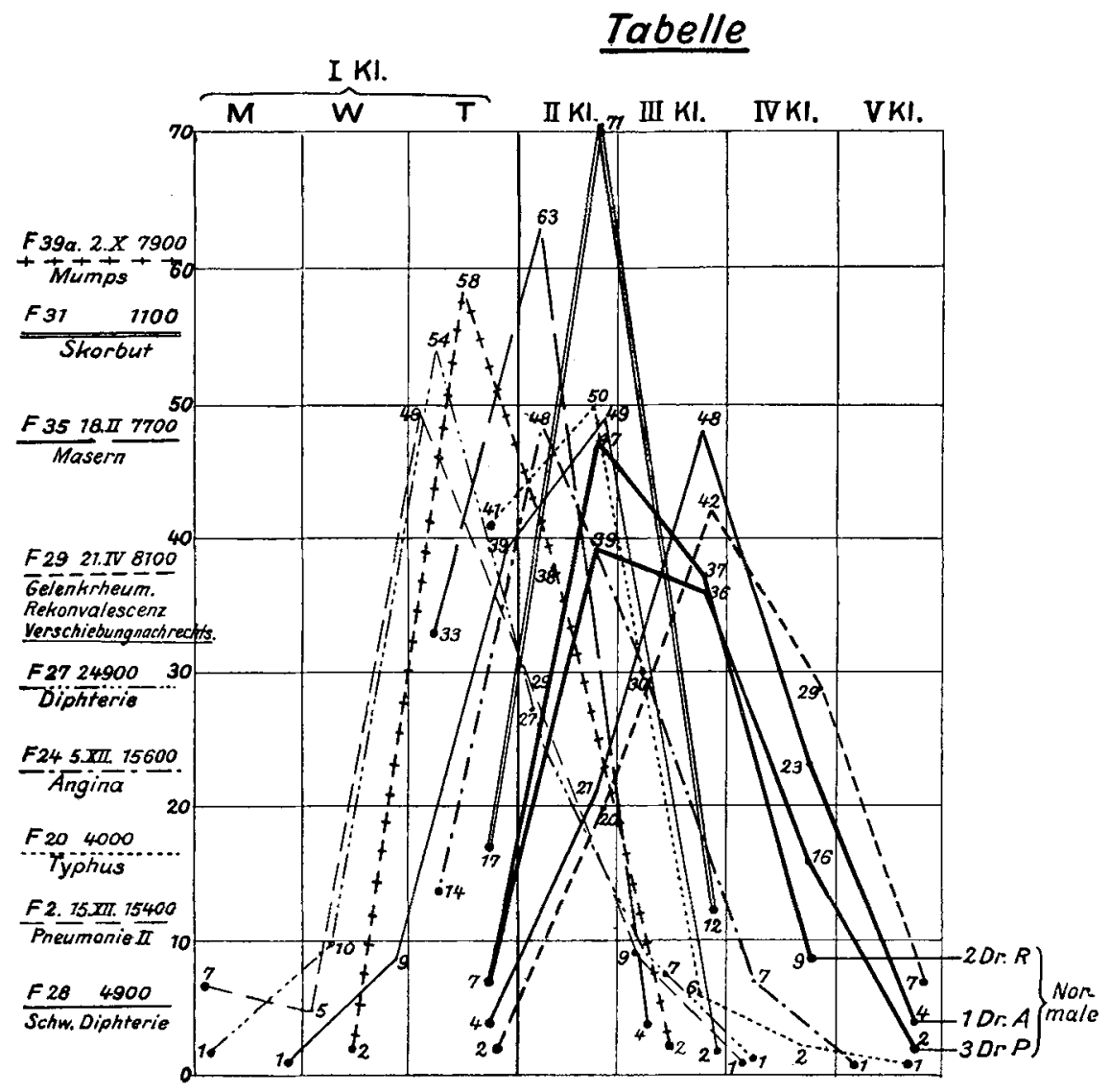

4. Die Arneth'sche 2.-5. Klasse wird unter Aufgabe der überflüssigen Unterabtheilungen in eine Gruppe von typisch-polymorphkernigen Neutrophilen als Segmentkernige zusammengefasst.

5. Die Klassen der Neutrophilen laten demnach:

a) Myelocyten, b) Jugendliche, c) Stabkernige, d) Segmentkernige. 
6. Dicsc noutrophile Eintheilung wird einfach eingereiht in das Differentialblutbild aller Leukocyten, so dass die übertriebene und specielle Beachtung des neutrophilen Blutbildes ausfällt. Ich crhalte demnach 8 Zellklassen:

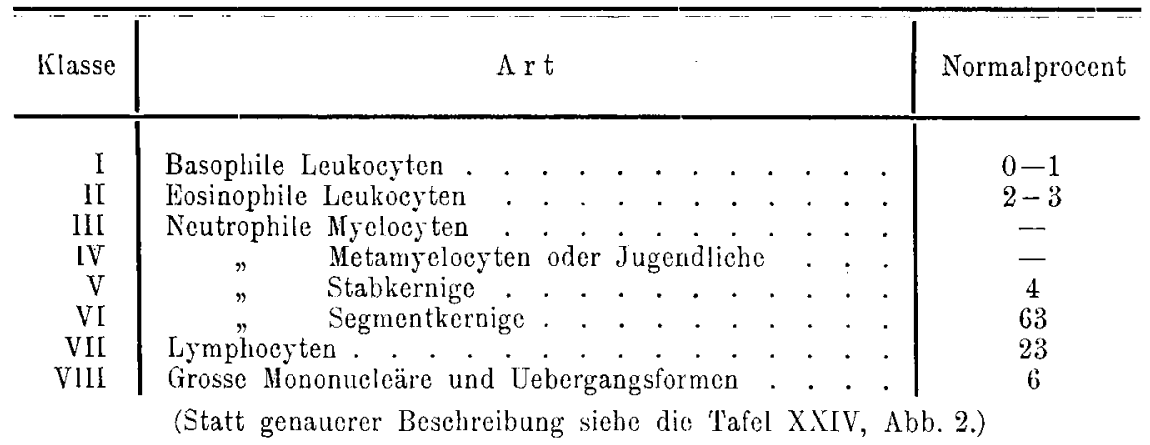

7. Auf Grund mehr als 2 jähriger Untersuchungen in zahlreichen Krankheitsfällen construirte ich einen Differentialleukocy tometer ${ }^{1}$ ) genannten $\Lambda$ pparat (s. Tafel XXIV, Abb.1), mit dessen Hülfe cine schnelle praktische Auszählung der mikroskopischen Präparate möglich war. Der A pparat liefert procentuale Jeukocytenbildor der unter 6 genannten Zusammensetzung, und stellt sie demonstrativ dar. Das kurz erwähnte Princip ist, dass 100 Stahlkugeln genau gleicher Grösse auf 8 den Zellarten entsprechenden Röhren nach dem Präparat vertheilt werden. Die Röhren sind so geaicht, dass das normale Blutbild einen horizontalen Strich, die Normale, bildet (s. Tafel XXIV, Abb. 1 und 3). Dadurch sind $A$ bweichungen nach unten und oben, Verminderungen oder Vermehrungen, gut ablesbar. Besonders deutlich markirt sich die Arneth'sche Verschicbung in den 4 Mittelröhren der Neutrophilen, da zwei normal leer sind (Ilyelocyten und Jugendliche), die dritte (Stabkernige) nur 4 pCt. höchstens enthält.

Ebenso deatlich markiren sich alle übrigen Veränderungen relativer Zusammensetzung: Lymphocytose, Mononucleose, Eosinophilie2).

Eine farbige Tafel gewährleistet, am Eussende des Apparates angebracht, möglichst gleichmässige Benutzung durch die mit dem A pparat Arbeitenden (genauere Beschreibung s. Schilling-Torgau 23).

Das Verdienst Arneth's ist es, durch eine genaue Methodik eine sebr constante Zusammensetzung der neutrophilen Zellklasse normal und eine gesetzmässige Veränderung der gesammten Zcllklasse pathologisch nachgewiesen zu haben. Wenn auch seine theoretisehe Erklärung nur für einen Theil der Fälle annebmbar bleibt, in den Nyclocyten und Metamyelocyten durch alle Uebergänge mit den reilen Formen verknüpft auftreten (Sepsis ctc., auch Leukämie), für andere Fälle, besonders Leukopenien (Typhus u. A.) aber eine von vornherein pathologisch und vermindert erfolgende Bildung angenommen werden muss, so wird doch dadurch das

1) Erhältlich bei Karl Zeiss, Jena, Geschäftsstelle Hamburg.

2) Kernhaltige Erythrocyten, Plasmazellen etc. werden in einer besonderen Rubrik des beigegebenen Notizblockes registrirt. 
Factum der Verschiebung selbst in keiner Weise berührt. Weit besser als durch die Verbrauchstheorie erklärt sich, wie gesagt, die Verïnderung des Blutbildes durch die dircete Bceinflussung der hämatopö̈tischen Organe.

Da dic Neutrophilen nun bei allen Jeukocytosen betheiligt zu sein pflegen, da in einer grösseren An\%ahl von Joukopenion jetht ebonfalls nachweisbar "Vorschiebungen" vorhanden sind, da wir ferner die übernormalen, normalen und subnormalen Zahlen nun durch ein von der Zahl unabhängiges Unterscheidungszoichen charakteristisch zu sondern vermögen, so ist m. li. der Werth der Feststellung der Arneth'sehen Verschiebung ein ganz ausserordentlicher, ja ganz unentbehrlicher. Wir dürfen zwar nicht erwarten, immer einen directen Maassstab für die Körperschädigung im ganzen zu erhalten, stets aber haben wir einen Anhalt für die Mitbetheiligung des Knochenmarkes durch Reiz oder Hemmung, die bei allen neutrophil-leukocytotactischen Krankheiten parallel bis fast gleich dem Gesammtzustande sein wird. Beziehen wir dieses neutrophile Bild in die gewöhnliche Differentialzählung ein und stellen es übersichtlich zusammen, wie ich es mit meinem Differentialleukocytometer versucht habe, so crhalten wir ausserordentlich verschiedene Typen von Blutbildern.

Es ist nach der Grösse des Gebietes noch nicht möglich, absolute Durchsehnittsbilder für cinzelne Krankheiten zu geben; dennoch hoffe ich, an diesen ausgewählten Fällen das Vorhandensein gewisser charakteristischer Gruppen gut erweisen zu konnen; der Hauptzweck der Arbeit aber ist, zu zeigen, wie sich droi wesentiche Factoren bei der Leukocytenbeobachtung erst diagnostisch, theilweise auch prognostisch, werthvoll ergänzen:

1. Die Gesammtzahl,

2. die Diffentialzusammensetzung,

3. die feinere Tellhistologie der Einzelklasse in der Arneth'schen Verschiebung.

\section{A. Normales und pseudo-normales Blutbild.}

\section{(Tafel XXIV, Abb. 3.)}

Die Normale ist nach dem Durchschnitt der Lehrbücher [EhrlichLazarus (24), Grawitz (1), Naegeli (2), Arneth (7) u. A.] construirt; dic Säulen bilden einen horizontalen Strich. Dic Zellklassen sind die gleichen der früheren Autoren bis auf die Neutrophilen, bei denen für das normale Blutbild die Rubrik Stabkernige hinzukommt. Auf Grund der von Arneth berechneien 5,2 pCt. Neutrophiler der 1 . Klasse und eigener Untersuchung berechnet sich ihre Zahl für $67 \mathrm{pCt}$. neutrophiler Zellen auf 3,5, abgerundet $4 \mathrm{pCt}$, im Gesammtlcukocytenbild, die in Röhre $V$ gesondert gezählt wurden.

Metamyelocyten sind Gelogenheitsfund. Myelocyten fehlen. Das normale Blutbild lautet also sammt den physiologischen Schwankungen: 


\begin{tabular}{|c|c|c|c|c|c|c|c|c|}
\hline $\begin{array}{c}\text { Nor- } \\
\text { males } \\
\text { Blutbild }\end{array}$ & $\begin{array}{l}I=B \\
\text { Baso- } \\
\text { phile }\end{array}$ & $\begin{array}{l}\mathrm{II}=\mathrm{E} \\
\text { Eosino- } \\
\text { phile }\end{array}$ & $\begin{array}{l}\mathrm{III}=\mathrm{MI} \\
\text { Myelo- } \\
\text { cyten }\end{array}$ & $\begin{array}{l}\text { IV }=\mathrm{J} \\
\text { (Metamye- } \\
\text { Jocyten) } \\
\text { Jugond- } \\
\text { liche }\end{array}$ & $\begin{array}{l}\mathrm{V}=\mathrm{St} \\
\text { Stab- } \\
\text { kernige }\end{array}$ & $\begin{array}{l}\mathrm{VI}=\mathrm{S} \\
\text { Segment- } \\
\text { kernige }\end{array}$ & $\begin{array}{l}\mathrm{VII}=\mathrm{L} \\
\text { Cympho- } \\
\text { cyten }\end{array}$ & $\begin{array}{c}\text { VIII = } \\
\text { Gr. M } \\
\text { Gr. Mono- } \\
\text { nucleäre }\end{array}$ \\
\hline $\begin{array}{l}\text { maximal } \\
\text { normal } \\
\text { minimal }\end{array}$ & $\begin{array}{c}(1) \\
1 \\
(0)\end{array}$ & $\begin{array}{c}(4) \\
3 \\
(2)\end{array}$ & $\begin{array}{c}(0) \\
0 \\
(0)\end{array}$ & $\begin{array}{c}(1) \\
0 \\
(0)\end{array}$ & $\begin{array}{l}(5) \\
4 \\
(2)\end{array}$ & $\begin{array}{c}(66 i) \\
63 \\
(61)\end{array}$ & $\begin{array}{l}(25) \\
23 \\
(21)\end{array}$ & $\begin{array}{c}(8) \\
6 \\
(4)\end{array}$ \\
\hline
\end{tabular}

Als Gesammtzahl der Leukocyten erseheint mir die Arneth'sche Berechnung von 5 bis 6000 für normale Lrwachsene besser zu stimmen als die früheren Angaben; sicher sind Werthe von über 8000 bei nüchternem Magen und ron unter 5000 bei uns pathologisch. Der Grund für scheinbare Abweichungen liegt meistens in latenten Krankheiten: Tuberculose Lues, Chlorose usw.

Sehr wesentlich ist, dass nach $A$ rneth und seinen Nachuntersuchern dic sogenannten physiologischen J eukocytosen in der Verdaung, nach Bädern, nach Muskelübung usw., anscheinend auch nach Blutverlusten, ohne Verschicbung verlaufen. Hier entscheidet die Gesammtzahl und meist vorhandenes Ueberwiegen von Neutrophilen im Differentialbilde. Während bei Arneth das neutrophile Blutbild gleich bleibt, sehen wir im Differentialleukocytometer sehon das relative Sinken der Lymphocyten und Steigen der Neutrophilen in Säule VI.

Ein sehr feiner Anzeiger des normalen Blutbildes sind ferner die Eosinophilen, die mindestens zu 2 pCt. vorhanden sein müssen. Ein Beispicl fast normalen Befundes ist Tafel XXIV, $4 b b .5 \mathrm{c} ; 9400$ Leukocyten, wo die fehlenden Eosinophilen mehr noch als die vermehrten grossen Mlononucleären den geübten Untersucher stutzig machen.

An demselben Blutbilde sieht man die praktisch werthlose ,Verschicbung nach rechts" Arneth's durch ein Absinken der Stabkernigen wenigstens angedeutet.

Es gicbt aber einzelne sehr scltene Fälle, die thatsächlich ein absolut normales Blutbild besitzen ohne Versehiebung und ohne Differentialänderung, bei denen allein die Zahl die Veränderung anzeigt. Einen reinen Fall konnte ich bei einem Falle von aplastischer Anänie in Folge chronischer Darmblutungen beobachten. Gerade wie die Erythrocyten allein eine dauernde Verminderung auf etwa 2000000 fast ohne pathologische Formen, wie Polychromasie, Megalocytose usw., aufwiesen, waren die Lcukocylen dauernd in normaler Zusammensetzung auf etwa 3000 wochenlang vermindert. Auf älnliche Fälle haben schon 'I'ürck-Helly (25) aufmerksam gemacht. Man bezcichnet sie am besten wohl als aplastische Hypolcukocy tose; vielleicht liegen auch ähnliche Verhältnisse bei der perniciosen Anämie für dic Leukocyten yor.

Zusammenfassend kann man sagen, dass nur die Blutbilder normal sind, die bei einer Zahl von 5-6000 und regelrechter Differentialzusammensetzung keine Verschicbung aufweisen. Pseudonormale Blutbilder sind nur an den Zahlverhälnissen zu erkennen. 


\section{B. Die Eosinophilie und Aneosinophilie.}

(Von einer leukocytären "Basophilic" sehe ich hicr ganz ab, dia praktisch wichtige Fälle bej Menschen unbekannt sind. Erhöhte Werthe werden z. B. bei der Leukämie eonstatirt. Der Vollständigkeit halber werden sie jedoch in Röhre I mitregistrirt.)

Die Eosinophilie registrirt sich"sehr deutlich in Röhre II. Dargestellt ist Tafel XXIV, Abb.4, ein Fall von ehroniseher, gutartig verlaufener Ankylostomiasis. Die Vermehrung gesehieht auf Kosten der Neutrophilen oft mit geringer Mononueleose.

Ausser Helminthiasis (4-6 pCt. schon verdächtig, wenn bei wiederholter Zählung gefunden) sind bekanntlich Hautkrankheiten, Pemphigus, Psoriasis, Urticaria usw., Heufieber, Neurosen und Asthma bronchiale Krankheiten, boi denen Losinophile vermehrt gefunden werden. Bei Puerperalerkrankungen soll Eosinophilie für gonorrhoische Aetiologie entscheiden [Lange (26)], auch die Cerny'sche exsudative Diathese soll durch Eosinophilie gekennzeichnet sein [Rosenstern (27)].

Differentialdiagnostische Bedeutung besitzt die Eosinophilie z. B. bei Tumoren für Echinococeus; bei Seliarlach entscheidet im Verlauf auftretende Eosinophilie gegenüber zweifelhaften Exanthemen, wie Masern, Röteln, Sepsis, Arzneimittel usw.

Die myeloischen Leukïmien gehen mit starken absoluten Vermehrungen der Eosinophilen einher, die sich allerdings relativ oft nicht direct ausprägen. Ein Beispicl ist Tafel XXV, Abb. 2, 1000000 Leukocyten mit 4 pCt. Eosinophilen; das bedeutet ein absolutes Ansteigen von 150 auf 4000 .

$\Lambda$ usführlicheres findet sich in den Lehrbüchern und den speciellen Arbeiten [Dunger (10), Stäubli (11)].

Viel Aufmerksamkeit ist in letzter Zeit dem Zustand des Fehlens oder der Verminderung der Eosinophilen geschenkt worden [Kownatzki(28), Blumenthal (29), Albrecht (30), Gräfenberg (31) u. A.].1)

Eine rolative Aneosinophilie findet sich, wie crwäbnt, bei sonst fast normalen Blutbildern als Unterseheidungsmerkmal.

Gerade bei leichteren Affectionen tritt oft schnell eine Rückkehr zur Norm ein, während die Losinophilen noch zögern (Tafel XXIV, Abb. 5 a bis 5 e, leichte Appendicitis). Andererseits kehren sie bei chronisehen Inlectionen oft vor der endgültigen Besserung des Blutbildes zurück und sollen daher gewissen prognostischen Werth bei der lïnschätzung der septischen Processe, z. B. von Pucrperalfiebern, besitzen. Sicher sind septisehe Infectionen mit Verbleib von Eosinophilen ('Tafel XXIV, Abb. $5 \mathrm{a}$-e) von vornherein günstiger zu bewerthen als fialle mit reiner Aneosinophilie ('Tafel XXV, Abb. 1a-0).

Die vorübergchende relative Aneosinophilie (mit günzlichom Fehlen nicht identisch, z. B. bei 0,1 bis 0,5 pCt.) ist ein Begleitzustand

1) Eine $\Lambda$ neosinophilie tritt auch durch Ueberreizung an die Stclle der Eosinophilie, wahrscheinlich infolge $\Lambda_{p}$ lasie, wie Stäubli für Trichinellen bei der Ratte experimentell zeigte; so erklärt sich vielleicht das Fehlen bei Botriocephslusanämie. 
des acuten Stadiums vieler Infectionskrankheiten, besonders des Typhus (Tafel XXVI, $\Lambda$ bb. 2-3). Sie findet sich auch bei schwerer Malaria in und kurz nach dem Anfall (Tafel XXV, Abb. 7, Tertiana, 1. Tag nach Chinin, 'lafel XXV, Abb. 8 'T'ropica, 2. Tag nach Chinin).

Unter den weiter hier abgebildeten Fällen findet sie sich als Nebenbefund bei einer schweren Pneumonie mit Benommenheit (Tafel XXVI, Abb. 7, 25000 Lcukocyten); bei so hohen neutrophilen Leukocytosen ist sie allerdings mehr relativ, obgleich hier in drei Tagen überhaupt keine Eosinophilen gefunden wurden. Das Gleiche gilt von einer schweren Syphilis mit Nephritis (Tafel XXVI, Abb. 6, 10600 Leukocyten) und von einer schweren Verbrennung mit nachfolgendem Erysipel (Tafel XXVI, $\Lambda$ bb. 5, 25800 Jeukocyten). Findet man also unter den gezählten Zellen mehrlach keine Eosinophilen, so überzenge man sich durch Absuchen des Ausstriches oder durch dic Zählkammermethode nach Dunger mit Färbung der Eosinophilen, ob sie wirklich ganz fehlen und notire lieber 1 pCt., wenn cinzelne vorhanden sind, als 0 pCt.

In den Sehlussbildern der Sepsisfälle (Tafel XXIV, Abb. 5iu.k und T'afel XXV, Abb. 1 a u. 10) fohlten die Eosinophilen in der That absolut in allen Präparaten. Wir sehen aber aus dem erst dureh cine Complication versohlechtorten Falle (Tafel XXIV, $\Lambda$ bb. 5) die bedingt prognostiseh günslige Bedeutung dor liosinophilen im Anfang und Verlaul bis $A b b .5$ h gut illustrirt (näheres siehe 'T'afelerkiärung).

Die Eosinophilie besitzt also eine unbedingt diagnostische Bedeutung für bestimmte Krankheiten, cine allgemeinere symptomatische und bedingt prognostische Bedeutung besonders für septische Infectionskrankheiten; ibro Mitregistrirung im Differentialleukocytenbilde ist daher unerlässlich.

\section{c. Die Veränderung der neutrophilen Lenkocyten und die Verschiebung des Blutbildes.}

Wegen der vorwiegenden Betheiligung der neutrophilen Kellklassen an fast allen Krankheitsprocessen und der an sich höchsten Zabhl dieser Zellen im relativen Blutbilde liegt naturgemäss hier der Schwerpunkt der meisten Leukocytenuntersuchungen. Im Differentialleukocytometer finden wir daher die 4 mittleren Sïulen für die Neutrophilen ihrer Bedeutung entsprechend reserviert.

Bei normalen Blutbildern und bei einer Reihe von Infectionen werden allein die Röhren V und VI, Stabkernige und Segmentkernige, benötigt. Fin grosser 'Theil der Verschicbungen verläuft in der. That ohne Auftreten von eigentlichen ansgeprägten dugondformon, sondern zeigt sich in einer Zunahme reifer, schlecht differonzirter, daher stabförmiger Kernfiguren (Beispiel: Tafel XXIY, Abb. 5a, leichte Appendicitis; Tafel XXV, Abb. 5, 6, 8, Malaria; Tafel XXVI, Abb. 1, Teberabscess; Tafel XXVI, Abb. 2 u. 3, Typhus; Tafel XXVT, Abb. 5, Erysipel und Verbrennung in Treilung).

Während im Arneth'schen Blutbilde von verschiedenen Autoren [Zelenski (19), Kothe und Sonnenburg (21) u. A.] die Zahl der 
ersten Klasse direct als Index benutzt worden ist, müssen wir hier im Differentialbilde eine klcine Umrechnung zur Feststellung des Grades der Verschiebung vornehmen. Der Verschiebungsindex ergiebt sich aus dem Verbältnis der Procentzahl der segmentkernigen Röhre VI zu den übrigen Neutrophilen Röhre III bis V. Der normale Index berechnet sich folgendermaassen:

$$
\mathrm{Jn}=\frac{63}{4}=13,2
$$

Ein pathologischer Index wäre z. B. Tafel XXV, Abb. 4, Malaria (Anfall):

$$
\mathrm{Jp}=\frac{40}{15+10}=\frac{40}{25}=1,6
$$

Man kann auch mit Umrechnung in absolute Zahlen den Versehiebungswerth bestimmen; es kommt hier der alte Streit über die Bedeutung relativer und absoluter Zahlenverhältnisse bei der Jueukocytose überhaupt in Betracht. Neuerdings sind erst Zangemeister und Gans (32) sehr energisch wieder für die alleinige Berücksichtigung absoluter Zahlenwerthe eingetreten auf Grund von Experimenten an Affen; der Kliniker wird jedoch wegen einer deutlich zu Tage tretenden gleichmässigen Betheiligung aller Blutzellen bei gutartigen Leukocytosen, ferner wegen des deutlichen Wechselverhältnisses zwischen Leukopenien und Lymphocytosen cinerseits und neutrophilen Hyperleukocytosen mit Lymphopenieen andererseits an der klinischen Bedeutung der relativen Verhältnisse festhalten müssen (Hecker (34), Kothe (35) u. A.). Wenn man allerdings mit einer einzigen Infectionsart arbeitet, wie Zangemeister und Gans, empfiehlt es sich, absolute Zahlen zu verwerthen, da hier nur das Verhältniss Leukocyten zu Lymphocyten graduell verschoben wird, nicht aber differential-diagnostisch in Frage kommt. Wir empfehlen also auch Berücksichtigung der absoluten Zahlon z. B. für Appendicitis allein oder puerperale Erkrankungen allein, halten sie aber differentialdiagnostisch für un übersichtlicher.

Die Festellung des Index der Verschiebung erscheint uns ebenfalls wenig wünschenswerth, da einerseits Höhe und Grad der Verschiebung individuell ausserordentlich schwanken und weder mit Virulenz des Erregers und Gesammtzustand des Patienten noch mit Verlauf und Prognose genügend harmoniren. Ohnehin lässt or sich bei einiger Uebung auch im Differentialblutbilde sicher genug durch einen Blick abschätzen, indem man das Verhältniss der Säulen III bis V vergleicht.

Nur in den Fällen, in denen die Leukocyten überhaupt geringere Bedeutung haben (Leukoponie mit Lymphocytose), erscheint die Verschiebung infolge des relativen Verhältnisses zu schwach registrirt, nähert sich aber der klinischen Bedeutung (Beispiel: Malaria, 'Tafel XXV, Abb. 5). Dagegen entspricht bei den neutrophil-wichtigen Krankheiten infolge relativnormaler oder hypernormaler Procentzahlen der Neutrophilen die relativ registrirte Verschiebung fast der absoluten Curve. Weit wichtiger als diese Gradunterschicdeder Verschiebung erscheinen uns Wesens- 
unterschiede, wie sie sich in dem Auftreten einerseits von Jugendformen ${ }^{1}$, andererseits von degenorativen Formen aussprechen.

Gorade wie bei den Zahlenverhältnissen die Höhe der Zahlen sich weniger brauchbar erwiesen hat als die Feststellung der llyperleukocytose oder Hypoleukocytose überhaupt, ist es auch bei der Verschicbung das Wichtigste, ob sio überhaupt vorhanden ist oder ob sie fehlt, ob die vorhandene Verschiebung durch degenerative oder missgebildete Zellformen oder durch jugendliche Reizungsformen des Markes hervorgerufen wird. Kliniseh ist z. B. die sehr schwere Leukopenie des Typhus mit rein degenerativem Typus belanglos gegenüber der sehwer regenerativen Erschöpfungsleukopenic der Sepsisfälle, die auf nahe Insuffizienz des Knochenmarkes deutet.

Die degenerativen Veränderungen der Neutrophilen finden sich sehr häufig mit leukopenien und relativen Iymphocytosen verbunden, d. h. Erscheinungsformen des Blutbildes, dic mehr auf Reizung des Jymphatischen Systems als des myeloischen hindeuten. Sie zeichnen sich durch lange Dauer, bei chronischen Erkrankungen oft viele Monate lang, vor den (bis auf dic Loukämie) vorübergehenden regenerativen Veränderungen aus, $\Lambda$ ueh dieser limstand erklärt sich weit besser durch Einflüsse auf dic Bildungsstätten als durch den Verbrauch. Experimentell im Thierversuch lïsst sich leicht erweisen, wie versehieden die Infektionserreger und ihre Toxine sofort auf das Blutbild einwirken, z. B. bei Sepsis einerseits und bei Typhus andererseits. Der uns klinisch bekannte Dualismus der weissen Blutzellen und eine geradezu gegensätzliche Beeinflussung des lymphatischen und des myeloischen Systems treten nach dem ersten Shock gewöhnlich sehr klar hervor. So verschieden aber der Grad dieser Reactionen beim Einzelindividuum, bei der Thierart, nach der Virulenz des Erregers ist, so constant ist der Typus der eintretenden Veränderung überhaupt für ganze Thiergruppen, für die einzelnen Zellklassen und für den Stamm der Infectionserreger.

Nach diesen theoretischen $\Lambda$ useinandersetzungen können wir versuchen, praktisch die Verschiebung selbst zu beobachten. Wir beginnen mit der Gruppe von Fällen, die die Theorien Arneth's noch am Besten rechtfertigen. Es sind dies die soptischen Erkrankungen, die bei nachweislichem Verbrauch von neutrophilen Segmentkernigen im Eiter eine constante durchgehende Verjüngung des Blutbildes neben degrenerativen Veränderungen erkennen lassen, eine lirscheinung, dic sich sicher am Besten durch die Schutzfunction der Leukocyten und vermehrte Reaction des Körpers erklären lässt. Allerdings ist dic rorwiegende Betheiligung der sogenannten älteren Kellen $\Lambda$ rneth's an der Schutzfunetion (Phagocytose) weder durchgehend im Blute noch in vitro zu constatiren [Pottenger (37), Busse (38)].

1) Eine grelegentliche Registrirung, wie sie die Klinili schon ror Arnoth kannte [s. die fast gleichzeitige Arbeit von Schindler (36)] und wie sie z. B. auch Papponheim wünscht ( $A$ tlas dec menschlichen Blutzellen), ist keineswegs ausreichend. 


\section{Tafel XXIV, Abbildung 5a--k.}

Der dargestellte Fall betraf einen jugendlichen, zuvor gesunden Mann mit typischen leichten $A$ ppendicitisbeschwerden; Leukocyten 10000 , T'emperatur $38,5^{\circ}$. Im Einverständniss mit dem Patienten wurde sofort zur Frühoperation geschritten.

1. Tag $\Lambda$ bb. 5a): L. 10000 zeigt vor der Operation deutliche Polynucleose (Säule VI) und sehr geringe Verschiebung (Säule V). Eosinophile normal (Säule II); relative Iymphopenic.

Die Blutveränderungen schlossen einen Abscess aus. Dic glatt verlaufene Operation ergab Endotyphlitis; an ciner Stelle Trübung der Serosa und beginnende Verfärbung der Mluseularis.

2. Tag (Abb. 5 b): J. 7500 , Temperatur $37,5^{\circ}$. Wohlbefinden; das Blutbild zeigt ausgeprägte neutrophile Polynucleose ohne Verschiebung, die in den nächsten Tagen (s. Tafelerklärung) bei dauernder Wundheilung und Wohlbefinden sich zur "Verschiebung nach rechts" ausbildete, d. h. mononucleäre neutrophile Elemente fehlen fast ganz. Gleichzeitig tauchen die Eosinophilen wieder auf (Abb. 5c und 5d). Dabci steigt die Zahl der Zellen resp. bleibt annähernd auf der Höhe von 12000.

Die Erklärung für diese dauernde Hyperlenkocytose, die in den ersten Tagen natürlich auf die Operation geschoben werden musste, zeigte sich am 5. Tage abends durch deutliche Schmerzen in der Wunde, die auch am 6. anhielten. Dementsprechend finden wir am 6. Tage (Abb. 5f) eine ausgesprochen regenerative Verschiebung, die bis zum 9. Tage gleichmässig ziemlich anhielt (Abb. $5 \mathrm{~g}$ ).

Diese hämatologisehen Befunde gaben Anlass zur sorglältigsten Sondirung der Wunde, die jedoch erst am 7. Tage einen kleinen Eiterherd in der Tiefe eröffnete.

Während der Folgezeit blieb klinischer und hämatologischer Befund bei leidlichem Wohlbefinden des Patienten gleich. Die offen gehaltene Wunde war andauernd belegt, lieferte viel Secret, und $a b$ und zu eröffneten sieh kleine Abscesseben in Wundtaschen.

(Beisp. 17. Tag (Abb. 5h), L. 13400.)

Klinische und hämatologische Befunde gaben bis zum 20. Tage keinerlei directen Anlass zur Befürchtnng, wobei vor allem auf die normalen Zahlen der Eosinophilen verwiesen werden soll, obgleich eine Wundinfection mit gutem Abfluss zweifellos bestand.

Leider fehlt der Blutbefund des 21. Tages, an dem gegen $\mathrm{Abend}$ peritonitische Erscheinungen plötzlich auftraten. Da es sich um eine ausgesprochene accidentelle Complication handelte, ist allerdings prognostisch kanm etwas zu erwarten gewesen.

22. T'ag (Abb. 5i): L. 33800; das Blutbild ist ein leukämoides, nur die Aneosinophilie (hier absolut) und die verhältnissmässig geringe Zahl von Myelocyten bilden noch einen Unterschied. Die Lymphopenie ist nicht nur relativ enorm tief, sondern absolut vorhanden.

Dieses Blutbild verhiess eine infauste Prognose, obgleich die klinischen Symptome mangels höherer Temperatur und mit Nachlassen der Schmerzen bis auf kleinen Puls weit besser erschienen. 
23. 'Tag (Abb. 5k): L. 25000; das Blutbild ist cin agonales und zeichnet sich durch das relative Fehlen aller übrigen Zellklassen, ausser den Neutrophilen, aus. Die Verschiebung ist unter Absinken der Zahl noch schwerer geworden; cin Zeichen, dass nach Arneth stets änsserst bedenklich ist. Abends: Tod.

Epikrise: Eine durchaus leichte Appendicitis wird durch Wundinfection, vielleicht schon während der Operation, complicirt. Die offene Wundinfection verursacht ohne stärkere Verbaltung nur eine mässige Reaction des sonst gesunden Körpers. Erst eine erncute Complication (Eröffnung eines latenten Eiterherdes mit hoch virulenten Erregern in die Bauchhöhle?) führt plötzlich unvorhergeschen zum Tode.

Die Beobachtung des Blutbildes zeigte diesen Verlauf parallel und theilweise klarer als der klinische Befund (2.-6. Tag). Prognostisch gestalten vor allem die Eosinophilen den Fall trotz doutlicher localer Sepsis günstig. Selbstverständlich war auch hier die Möglichkeit einer mehr äusserlich begründeten Complication ins Auge zu fassen, ihr Eintritt aber nicht nothwendig, zufällig und nicht voraussagbar. Die hohe Leukocytose der letzten Tage, dio bei alleiniger Beachtung der Zahl, besonders auch mit dem Absinken, vielfach günstig gedeutct wäre, und sich auch wohl nur durch die bosonders hohe Reactionsfähigkeit des krä̈tigen jungen Patienten crk]ärt, erseheint im Differentialblutbilde mit Arneth'scher Verschiebung trotzdem sofort als gänzlich infaust.

\section{Tafel XXV, Abbildung $1 \mathrm{a}-\mathrm{f}$.}

Ein zwciter vollständig wiedergegebener Fall diene zur Illustration der Verschiebung vom septischen Typus bei einer reinen, von vornherein bösartigen Sepsis.

Der Befund ist folgender: Der ebenfalls junge kräftige Patient zog sich eine leichte Scheuerwunde am Knöchel zu. Die vernachlässigte Wunde begann am 3. Tage zu schmerzen; unter Verdacht auf Blutvergiftung wurde Pat. sofort in klinische Behandlung geschickt.

Die kleine Scheuerwunde am linken Knöchel zeigte sich etwas grau belegt in teigig geschwollener Umgebung ohne stärkere Röthung. Am Unterschenkel zarte röthliche Streifung, entsprechend den Lymphgefïssen. Leistendrüsen schmerzbaft geschwollon. Sofort ausgedehnte Incision. In bezug auf die genaueren Blutbefunde sei auf die Tafelerklärung verwiesen.

Auch hämatologisch gab von Anfang an die geringe Leukocytose, die zunehmend noch absank und subnormale Werthe erreichte, sowic die Aneosinophilie und dor Tiefstand der Lymphocyten zu crnsten Bedenken Anlass. Besonders am 3. Tag (Abb. 1e) gestaltet die zunehmende Verschiebung bei Absinken der Zahl die Prognose schon sehr ungünstig. Das ausgesprochen schwer degenerative Blutbild des 4. Tages (Abb. 1d) ist vielleicht mit dem weiteren Sinken der Zahl als eine Art Aplasie aufzufassen. Die absolute Lympliocytenzahl sinkt auf 355 , statt 1320 normal. Es tritt ein von Gräfenberg (31) für sehr wichtig gehaltenes Phänomen ein: die Curve der Lymphocyten kreuzt die 
sonst niedrigere Curva der grossen Mononucleären. Prognose infaust. Klinischerseits wurden die ständig verlängerten Incisionen nicht mehr für ausreichend gehalten und die Amputatio cruris vorgenommen; deutliche Infiltrationen in der Tiefe veranlassten die sofort angeschlossene Amputatio femoris.

Der 5. Tag (Abb. 18) zeigt das Blutbild zwar, besonders in dem Jymphocyten und Mononucleären etwas gebessert, dennoch ist bei der abnormen niedrigen Zahl (5100) der Befund hoffuungslos. Es ist eine typische Anisohypocylose Arneth's.

Der 6. Tag zeigt Morgens 10 Uhr ein agonal leukämoides Blutbild (Abb. 1f). Abends: Tod.

Epikrise: Von vornherein sebr ungünstig zu beurtheilende Sepsis durch Erreger hoher Virulenz (bakteriologisch: hämolytische Streptokokken in langen Ketten). Hämatologisch dementsprechende sofortige schwere Verschiebungen bei verhältnissmässig geringen Leukocytenzahlen, Aneosinophilie und absolute Lymphopenien. Das agonale Blutbild mit agonaler Hyperleukocytose ist im Sinne Arneth's ein Krankheitssymptom, nicht etwa cin eigentliches Todessymptom, und beruht auf gänzlicher Insufficienz des Markes.

Diese beiden Fälle, die sich aus meinem Material um viele vermehren liessen, zeigen mit absoluter Deutlichkeit die hohe Bedeutung eines vollständig aufgenommenen Blutbildes bei septisch-chirurgischen Erkrankungen. Der Typus der Verschiebung ist eine so ausgesprochene Neutrophilie mit Regeneration, dass auch die differential-diagnostische Verwerthbarkeit in allen Fällen von Infectionskrankheiten, bei denen erst septische Complication das regenerative Moment in das Blutbild hineinträgt (z. B. Typhus, chronische Erkrankungen wie Tuberculose, Syphilis etc., Protozoenkrankheiten, aber auch aseptische Operationen, Wochenbett etc.) un$z$ weifelhaft im höchsten Maasse erwartet werden darf. Die prognostische Bedeutung ist mehr eine allgemeine, insofern echte Complicationen nie ausgeschaltet werden können, und der Eintritt der Veränderungen meist auch nur wenig dem klinischen Befunde vorauseilt. Dennoch gestatten, wie Fall XXV lehrt, Beharren und Form der Verschiebung oft werthvolle Schlüsse auf die Tendenz des ganzen Krankheitsprozesses zur eventuellen Verschlimmerung.

Im Folgenden sollen einige weitere Beispiele von Blutbildern gegeben werden, bei denen dio Neutrophilen die charakteristischen Veränderungen zeigen. Ich kann mich dabei nur auf ausgewählte Fälle beschränken, habe mich jedoch bemüht, möglichst typische Bilder auszusuchen. Hinsichtlich der Infectionskrankheiten muss betreffs der Arneth'schen Verschiebung von vornherein auf die Monographie Arneth's (16) bingewiesen werden, die das wesentliche in grosser Breite enthält (s. auch Curven S. 692).

In den Schlussbildern der Sepsisfälle hatten wir leukämoide Blutbilder kennen gelernt, die wir jetzt mit echt leukämischen vergleichen wollen. 
Die loukämischen Blutbilder sind wegen ihrer intercssanten Zusammensetzung viel studirt worden, dennoch ist eine genaue Analyse erst seit der Röntgentherapie auch praktisch bedeutungsvoller geworden. Arneth $(39,40)$ hat seiner Methode hicr den schönsten theoretischen Erfolg zu verdanken; er konnte beweisen, dass die Besserung des leukämischen Blutbildes unter den Röntgenstrahlen nicht durch Zerstörung der pathologischen Zellen, sondern vor Allem durch einen indirecten Einfluss auf die hyper- und metaplastische Markthätigkeit zu erklären sei; nicht nur cine Verringerung der \%ellzahl, sondern eine bessere Ausreifung der Einzelform zeigte sich auf's Deutlichste. Uebrigens erklärt sich hier $\Delta$ rneth ganz gegen seine sonstigen Ideen die Abhängigkeit auch der '/ahl nicht vom Verbrauch, sondern allein von der Knochenmarksbildung, die Gesammtkerngestaltung ebenso nicht abhängig von einem verminderten Verbrauche älterer /ellen, sondern von besserer Ausbildung bzw. Herabminderung der Ausschwemmung jugendlicher Zellen.

Tafel XXV, Abb. 2 und 3 zejgen solche myeloiseh-leukämische Blutbilder bei 100000 und 60000 Gesammtzahl der Ceukocyten.

Ohne Weiteres fällt die colossalo Verschiebung in die jugendlichen Zellklassen ins $\Lambda$ uge. Charakteristisch ist der Abfall von den echten Jugendformen über die Stabkernigen zu den Segmentkernigen. Ein wesentlicher Unterschicd gegenüber den septisch-leukämoiden Bildern ist das Vorhandensein der Eosinophilen trot\% der enormen Hyperleukocytose. Die absolute Vermebrung muss hier riesig sein, um Schritt halten zu können (normal bis 180, hier 4000 und 1200 Eosinophile).

Die Verminderung der Lymphocyten ist relativ. Ihr wirkliches Verhältniss lässt sich nur mit sehr umfangreichen Zählungen feststellen und hat nur wissenschaftliches Interesse.

Die hohe Zahl der grossen Mononueleären wird besonders im zweiten Falle durch isomorphe Agranulocyten, d. h. ganz unreife Myelocyten ohne Körnung (Myeloblast Naegeli's) hervorgerufen.

Bekanntlich können diese Myeloblasten lymphatische Teukämien vortäuschen. Oft hilft hicr noch die khrlich'sche Triacidfürbung, wenn May-Grünwald und Giemsa schon versagen, um die letzten feinen Granula festzustellen. Auch die proteolytische Fermentprobe (Jochmann und Müller), die Oxydasereaction (Winkler u. A.), kann herangezogen werden, versagt aber oft gerade in Grenzfällen. Die Schriddeschen Lymphocytengranula sind nicht constant genug färbbar und auch nicht absolut specifisch. Die Beobachtung von echten Uebergangsformen der Polynucleären zu den Myelocyten in der Mrnethschen Weise ist also wohl brauchbar, um die Diagnose zweifelhafter Fällo zu unterstützen.

(Blutbilder von lymphatischer Leukämie sind ausserordentlich eintönig und wegen der hohen Procentzahl der Lymphocyten im Differentialleukocytometer ohne Umänderung der Bezeichnung nicht darstellbar.)

Arneth $(39,40)$ hat die Anwendang seiner Methodik bei der Röntgenbehandlung myeloischer Leukämie gezeigt. Nicht das Sinken 
der hohen Gesammtzahl allein ist ein Zeichen der Besserung, sondern erst eine damit Hand in Hand gehende Verringerung der Jinksverschiebung. Auch hier wurden fast normale Zahlen mit schwerster Verschiebung und ebensolche mit fast völliger Restitution des normalen Blutbildes beobachtet. Dio Beobachtung der Verschicbung kann also einen vorzüglichen Indicator für dic Dosirung der liöntgenstrahlen bezw. Abbruch der Behandlung ergeben. Mangels cigener Fälle verweise ich auf die Literatur $[\Lambda$ rneth $(39,40)$, Franke (41), funck (42) U. A.].

Theoretisch recht interessant sind die Verschiobungen bei Malaria, die jüngst Gothein (43) nach der Arneth'schen Methode studirte. Er fand in und kurz nach dem Anfall während der Anfallsleuko(ytose und vor der folgenden Leukopenie ${ }^{1}$ ) deutlichste Verschiebung. Bei frühen Recidiven will or das Fortbestehen einer leichten Verschiebung bis zum erneuten Anfall und in diesem cine Cumulation beobachtet laben. Das Arneth'sche Blutbild hat daher eine gewisse prognostische Bedeutung für Recidive; weiter soll es diagnostisch besonders bei eintretenden Complicationen verwerthbar sein.

Diese Befunde decken sich gut mit eigrenen unabhängig gemachten; allerdings gehört Malaria m. E. zu den Erkrankungen, bei denen die Neutrophilen Nebenbefund sind. Im Anfall werden zwar, besonders bei schweren Fällen, vereinzelte Myeloryten und immer Metamyelocyten gefunden; der Befund weehselt aber oft enorm schnell. In der Leukopenie treten dann degenerative fiormen spärlich in den Vordergrund. Das Charakteristische liegt in den grossen Mononucleären.

Tafel XXV, $\Lambda b b .4$ und 5 zeigt den gleichen Fall von T'ertianarecidiv, in der Anfallshyperleukocytose mit regenerativer Verschicbung und am nächsten Tage unter der Chininwirkung bereits mit Leakopenic und absoluter Lymphocytose und Mononucleose. Diese Anfallshyperleukocytose ist wohl wie die experimentelle Anfangshyperleukocytose mehr eine plötzliche Aussehwemmung auf den infectiosen Insult hin, als eine wirkliche hyperplastische oder regenerative Produetion.

Dic $\Lambda$ bbildungen 6 und 7 zeigen ebenso wie 5 , dass das Hauptcharakteristicum eben bei den lymphocyten und grossen Mononueleären zu suchen ist, dass aber gleich nach dem Anfall eine für die Tahl der Neutrophilen ausserordentlich hohe Verschiobung besteht (Verhältniss der Röhre VI gegen 111 bis V). Dabei ist es gleichgültig, ob die Parasiten noch im Blute kreisen. Abbildung 6 und 7 sind beides Bilder vom Tage nach dem Anfall unter Chinin, 6 mit Parasiten, 7 ohne Parasiten. Abbildung 8 von einer Tropica 2 Tage nach dem Anfall zeigt schon den Anstieg der neutrophilen Sogmentkernigen mit Abfall der Verschiebung. In diescm Falle blieb jedoch, da es sich um einen stark recidivirenden Fall handelte, das 13lutbild in dieser Form constant, wie folgende Tabelle zeigt.

1) IJiese bekannte Leukopenie scheint bei Malaria keineswegs absolut typisch zu sein; augenblicklich am Instituł ausgcführten Untersuchungen ergaben meist Hyperleukocytosen. 


\begin{tabular}{l|c|c|c|c|c|c|c|c|c}
\hline & B. & L. & M. &. & S. & St. & I. & Gr. M. & $\begin{array}{c}\text { Gesammt- } \\
\text { zahl }\end{array}$ \\
\hline 1. Tag: Anfall & - & - & - & 1 & 26 & 35 & 27 & 11 & 7100 \\
2. " Chinin & - & 1 & - & 2 & 16 & 32 & 35 & 14 & 6900 \\
3. " & 1 & - & -- & - & 6 & 21 & 36 & 36 & 3900
\end{tabular}

usw. langsame Zunalime auf 4500; Verschiebung constant 6-10; dauernd reichlich Halbmonde. Nach Gothein also Reeidivgefahr. Patient wurde dann cntlassen.

In geheilten Fällen geht dagegen das Blutbild (bei den Lymphocyton und grossen Mononucleären allerdings sehr langsam) zurück, während dic Neutrophilen mit ausgesprochener "Versehiebung nach rechts", d. h. in unserer Methode abnorm nicdrigen Zahlen der Stabkernigen, sich den normalen Werthen der Neutrophilen langsam nähern. $\Lambda \mathrm{m}$ Schluss der Krankenhausbehandlung sind Bilder wie Tafel XXV, Abb. 9 (Leukocyten 6200) die Regel.

Genauere Beschreibung der Blutbilder siche Tafelerklärung und letzten Abschnitt.

Drei im Typus zuerst sehr ähnliche Verschicbungen zeigt die Tafel XXVI, Abb. 1-3. Alle drei sind ausgesprochen degenerativ. Die Sonderung ist aber nach den Befunden in den übrigen Zollklassen leicht $\mathrm{möglich}$.

Tafel XXVI, Abb. 1, L. 5900; Verdacht auf Juberabscess. Der aus den Tropen kommende Patient war schon wiederholt an Jeberabscessen erkrankt und bereits operirt. Augenblieklich bestand nach längerem Intervall eine durch Schmerzen und unregelmässige Temperatursteigerungen gekennzeichnete acute Erkrankung, die jedoch einen sicheren Befund auch dureh Punction nicht gestattete.

Der Fall ist eine schöne Illustration der Bedeutung der Arneth'schen Verschiebung. Sowohl die Cresammtzahl sowie die relative Zusammensetzung der Zellklassen ist ohne Rücksicht auf die Verschiebung fast normal. Die Verschiebung registrirt jedoeh sehr deutlich die bestebende septische heizung. wahrscheinlich nur durch 'loxine. Da in Zukunft sich der Zustand des Patienten besserte, wurde von ciner Operation abgesehen.

Achnliche einfache Verschicbungen bei nur geringer Hyperleukocytose (zwischen 8-10 000) wurden gerade bei Leberabseessen bäufig beobachtet und erklären sich viclleicht, wie es auch bei Appendicitis bekannt ist, durch die gute $\Lambda$ bkapsclung der Abscesse ${ }^{1}$ ).

Dic Versuche, aus dem Leukocytenbefunde auf Eiter direct zu sehliessen, sind auch theoretisch als völlig verfehlt anzusehen. Nicht der bestehende kitor, sondern die Grände \%u seiner Bildung verursachen die Loukocytose; also werden wohl Anfänge und Fortschritte, niemals aber Zustände der Vereiterung registrirt.

1) Die St-Zellen dieser und ähnlicher Fälle zeigen das Aussehen der auch normal vorkommenden seltenen St-Formen, sind also nicht wirlilich degenerativ: derartig feine Unterschiede lassen sich vorläufig nicht gut festhalten. 
Das Blutbild XXVI, Abb. 1 , ist der Typus einer leieht neutrophilwirksamen $\Lambda$ ffection und würde in diesem Falle den augenblicklichen Fortschritt eines vielleicht chronisehen Abscesses bedeuten.

Tafel XXVI, Abb. 2, J. 4000 und Abb. 3, J. 3000, sind Typhusfälle der 3. Woche, mit typischer leukoponie, Aneosinophilie und relativer Lymphocytose. Die Verschiebung ist charakteristisch degonerativ und erklärt sich am Besten nach Naegeli mit Knochenmarkshemmung. Obgleich der Nachdruck auch hicr mehr im lymphocytären Bilde und der Leukopenie liegt, gegenüber den septischen Blutbildern (Abb. 1), so sondert dic Verschicuung dieses Blutbild schön von Blutbildern des gleichen Typus bei chronischen Ernährungskrankheiten (Beriberi, $\Lambda$ bb. 4), Jseudoleukämien, Banti usw.

Wichtig ist vor allen Dingen bei lymphocytären Blutbildern immer die prompte Anzeige einer septischen Complication; als Beispiel setze ich hier dic Aufzeichnung eines Typhus mit Complication durch Pyelitis her:

$$
\begin{array}{ccccccccc}
\text { Zahl } & \text { B. } & \text { E. } & \text { M. } & \text { J. } & \text { St. } & \text { S. } & \text { L. } & \text { Gr. M. } \\
13000 & - & - & -- & - & 9 & 77 & 9 & 5
\end{array}
$$

Es besteht ausgesprochene Neutrophilie mit so geringer Verschicbung, dass hier die von Arneth erwähnte antagonistisch wirksame Verbindung zweicrextrem entgegengesetzter Factoren bzw. ihrer Blutbilder ganz plausibel erscheint.

Als besondere Fälle seien noch erwähnt: TafelXXVI, Abb.7, Leukocyten 24800 (genauere Beschreibung siehe T'afelerklärung); Pnoumonie.

Charakteristisch für Pneumonie ist die hohe Leukocytenzahl und die breite (mehr septische) Verschicbung, dic bei guter Reaction des Körpers stets zu finden ist. Die Lymphopenie ist relativ; die Prognose lautet in Anbetracht der geringen Myelocytenzahl und der hohen Mononucleose günstig. Schon zwei Tage später findet sich folgendes Blutbild:

$\begin{array}{ccccccccc}\text { Zahl } & \text { B. } & \text { E. } & \text { M. } & \text { J. } & \text { St. } & \text { S. } & \text { L. } & \text { Gr. M. } \\ 29500 & - & - & - & 3 & 26 & 47 & 18 & 6\end{array}$

(1)

Die Lymphocyten sind wesentlich nachgekommen in der relativen Tahl, die Jugendzellen haben abgenommen, Prognose sehr günstig, da die Reaction übercompensirt geleistet wird. Ausgang: Heilung.

Tafel XXVI, Abb. 6; schwere Lues mit Nephritis, 1. 10600.

Schr gefährlicher Allgemeinzustand; drohende Anurie; Cylindrurie. Blutbild zeigt hohe Neutrophilie mit ganz unverhältnissmässig geringer Verschiebung; Sepsis und Pneumonie ausgeschlossen.

Dies Blutbild gehört zu der Gruppe von Krankheiten, bei denen das Blutbild vorläufjg wenig Charakteristisches zu bieten scheint. Die Veränderungen der inneren Organe, wie Niere, Leber usw., soweit sie ohne acute auf das Blutbild einwirkende Infection entstehen, wirken durch die Functionsschädigung sehr auf den Allge- 
meinzustand, wenig auf das hämatopoetische System. Sie fallen also in ihrem klinischen Werth stark gegen die Blutbilder der hier sonst behandelten Krankheiten ab, obgleich bei dauernder Beobachtung des Blutbildes im Verlauf eines Einzelfalles gewisse symptomatische und prognostisch vielleicht verwerthbare, dem Allgemeinzustand parallel verlaufende geringe Veränderungen nachweisbar sind.

\section{Tafel XXVI, Abb. 5, L. 25000.}

Dieses Blutbild stammt von einem heilenden Erysipel, das von ausgedehnten Verbrennungswunden seinen Ausgang nahm, und zeigt, wie wenig selbst ausgesprochen neutrophil-leukocytotaktische Processe zu wirken vermögen, wenn die Allgemeininfection bzw. Intoxication durch die Oberflächlichkeit des Processes verhindert wird. Prognose und Verlauf war dementsprechend sehr günstig.

Aehnliches hatte schon der Sepsisfall (Tafel XXV) gezeigt.

Betreffs weiterer Ausführung über die Bedeutung der Verschiebung muss ich auf die Specialarbeiten und die Literatur hinweisen. Sowohl für Gynäkologie als Chirurgic liegen schon zahlreiche Arbeiten vor [siehe Schilling-Torgau (44), Sammelreferat]. Sehr werthroll scheinen nach den Specialstudien, die von Arneth (45) inaugurirt wurden, die Ergebnisse in der Tuberkuloseuntersuchung zu sein. Die Anwendung der Verschiebung als feinstes Reagens besonders auf Tuberkulininjection, Bestimmung des Zeitpunktes der Injection nach Abklingen der Verschiebung, die Bewerthung der eingotretenen Heilung nach dem Grade der Rückkchr zur Norm, alles das sind nachgeprüfte und grösstentheils bestätigte Thatsachen, die hier nur erwäbnt werden können [Arneth (45), Rövor (46), Uhl (47), Dluski und Rospedzihowski (48), von Bonsdorff (49) u. A.].

Die Zusammenfassung unserer Beobachtungen mit der Eintheilung des Differentialleukocytometers sind kurz folgende:

1. Die Arneth'sche Verschiebung lässt sich bei Fällen mit Neutrophilie einwandsfrei, in den übrigen ausreichend im Rahmen des Differentialgesammtbildes beobachten.

2. Höhe und Grad der Verschiebung ist diagnostisch und prognostisch wegen individueller und sonstiger Schwankungen wenig brauchbar; bei fortlaufender Beobachtung eines Falles oder vergleichender Beobachtung zahlreicher Fälle der gleichen Krankheit ist ein gewisser Parallelismus mit dem Gesammtzustande zu constatiren.

3. Differentialdiagnostisch lässt sich nur der Gesammttypus des Blutbildes incl. der Verschiebungsform, ob regenerativ und pathologisch oder nur pathologisch degenerativ, vortheilhaft gebrauchen.

4. Die prognostische Bedeutung fällt fast stets zusammen mit der augenblicklichen symptomatischen Bedeutung. 
5. In Fällen ohne specifische Verschicbung kann der Eintritt einer solchen als werthroller Anzoigor einer Complication benutzt werden; andererseits kann eine an sich uncharakteristische Verschiebung ein wesentlichor Factor für die difforential-diagnostische Einsehätzung sonst gleicher Blutbilder der anderen Zellklassen werden.

\section{Die Lymphocytosen und Mononucleosen.}

Vieles Hierhergehörende ist bereits kurz erwähnt.

Im Allgemeinen pflegen Protozoenkrankheiten, chronische Affection ohne Complication ('Tuberculose, Lues), eine Reihe ätiologisch noch ungeklärter Krankheiten wie Banti, klinische Pseudoleukämien, Milztumoren aller Art [Hess (5) u. A.], viele maligne uncomplicirte Tumoren, Basedow, Basedowoid mit gering erhöhten Gesammtzahlen oder subnormalen und direct chronisch-leukopenischen Worthen zu verlaufen. Meistens findet sich dabei eine absolute oder relative Lymphocytose und Mononucleose.

Genaue Erforschung dieses Gebietes scheitert bisher an zwei Umständen: der mangelhaften Eintheilung der Jymphocyten wegen fehlender Merkmale und der unklaren Stellung des grossen Mononueleären und der sogen. Uebergangsformen. Dennoch sind auch hier durch genaueres Studium Fortschritte zu erwarten und erreicht [Hess (5), Fabian (6 u. 7), Benjamin (4), Kocher (8), Caro (9) u. A.]

Wir wollen hier nur einige typische Formen der Veränderung geben, deren Worthung und Wiederkehr auf Specialgebieten anderen Studien vorläufig überlassen bleiben muss.

Wir haben die relative Lymphopenie als symptomatischen Factor bei der Beurtheilung septischer Processe schon kennen gelernt. Ein gutes Schritthalten der Lymphoeyten mit der Leakocytose ist sicher ein günstiges Zeichen [BI umental (29), A lbrecht (30), Gräfenberg (31) u. A.]. Auch die Nachholung eines Verlustes ist prognostisch günstig zu werthen (Tafel XXIV, Abb. 5b und 5c).

Dagegen ist starkes Absinken (Lymphocytensturz!) cin allbckannten böses Omen (Tafel XXIV, Abb. 5 und Tafel XXV, $\Lambda$ bb. 1, Schlussbilder), das in jüngster Zeit viel beachtet ist [Albrecht (30), Gräfenberg (31), Busse (50), Sondern (51) u. A.). Die "Kreuzung der Nononucleärencurve" [Gräfenberg (31)] ist als Gradmesser nicht uninteressant, obwohl nicht bei allen Krankheiten gleich bodeutend ungünstig; z. B. tritt sie ohne böse Bedeutung bei Mononucleosen schr loicht ein.

Die infectiöse Lymphocytose mit gleichzeitiger Ver. minderung der Neutrophilen ist $\%$. B. beim Typhus (Tafel XXVI, Abb.2 und 3) relativ sehr ausgesprochen, und wird in Reconvalecenz meist absolut.

Diese Form ist von der relativen und absoluten Lymphocytose chronischer Krankheiten der oben erwähnten dunklen Aetiologie durch geringere Reaction der grossen Mononucleären und durch das Vorhandensein einer ausgesprochenen Ver- 
schiebung der Neutrophilen getronnt; bei den chronischen Erkrankungen findet sich dagegen manchmal statt der sonst sehr geringen "Verschiebung nach links" eine "Verschiebung nach rechts".

Tafel XXVI, Abb.4. L.5400. Beriberi. Beispiel einer hohen absoluten Lymphocytose mit deutlicher Mononucleose und fast fehlender Verschiebung. (Derartige Blutbilder sind jedoch nicht typisch für Beriberi; die Zusammensetzung ist oft auch fast normal, ja leichte Verschiebungen kommen vor. Genaue Untersuchungen sind noch selten.)

Bei Protozoenkrankheiten (Malaria, Kala-azar, Trypanosomiasis) pflegen die Blutbilder ähnlich zu sein, nur in den Grossen Mononucleären bekanntermaassen erheblich zu überwiegen (Malaria, TafelXXV, Abb. 5-8). Im Anfall tritt bei Malaria einfache Verschiebung (Tafel XXV, Abb. 6 und 7 ) oder directe Ausschwemmung jugendlicher Elemente mit Verschiebung auf (TafelXXV, Abb.4). Dennoch bleibt speciell die Mononucleose leicht sofort oder im Verlauf erkennbar.

Die pseudoleukämischen Granulomatosen (Tuberculose, Lues) zeigen besonders im entzündlichen fortschreitenden Stadium ausgesprochene mässige neutrophile Polynucleose mit geringer Verschiebung [Arneth (16), Fabian (7) u. A.], ähnlich wie Carcinome und Sarkome fortgeschrittener Art [Baradulin (52) u. A]. Demgegenüber sollen dic echten Pseudoleukämien oder bessor aleukämischen Leukämien ausgesprochene relative oder absolute Lymphoeytose bei meist niedriger Zahl haben (Pinkus'sches Zeichen), nach Arneth ohne Verschiebang.

Der Streit um die differentielle Bedeutung der Lymphocytose bei Basedow und Basedowoid gegen Struma [Kocher (8), Caro (9) u.A.] ist noch unentschieden. Alle diese Befunde sind wegen ihrer geringen Ausgeprägtheit schwer unterscheidbar.

In interessanter Weise hat Benneke jüngst versucht, allein nach der Leukocytose Scharlach als Mischinfection cines lymphocytär-wirksamen unbekannten Erregers und eines septischen leukocytär-wirksamen Streptococeus zu erweisen, da sich ähnliche Leukocytosen bei anderen Mischinfectionen, z. B. Variola, Tuberculose usw. vorfinden, resp. ein ähnlicher aber zeitlich getrennter Verlauf der Ljeukocytenveränderung bei Masern mit Complication zu beobachten ist.

Die Mononucleären allein sind erst von wenigen Forschern ernstlich der Diagnostik dienstbar gemacht worden, doch sind sie sicher nicht ohne Bedeutung, da wir sie im Thierexperiment wichtige Functionen als Makrophagen verrichten sehen [Rieux (53) u. A.). Die Patella'schen Hypothesen (54), die sich auf die endotheliale und angeblich nekrotische Natur der Mononucleären stützen, wollen ihr Auftreten mit Gefässalteration erklären, sind aber theilweise in dieser Form unhaltbar, obgleich dic klinischen Beobachtungen mit anderer Erklärung stimmen dürften.

Ein gewisser Parallelismus mit den Neutrophilon scheint in Sepsisfällen oft vorhanden zu sein, obgleich bei schwerer Sepsis eine ausgesprochene Verminderung auch der Mononucleären mit den 
Lymphocyten erfolgt. Vicl ausgesprochener sind Lymphocytosen mit Mononucleosen gepaart (z. B. Typhus, Beriberi). Tafel XXVI, Abb. 2-4.

Endlich giebt es auch Fälle mit selbstständiger Mononucleose z. B. alte Malaria, Amöbiasis [Rieux (53)] ${ }^{1}$ ). Im Ganzen ist also das Verhalten der grossen Mononucleären ein Beweis für ihre eigene Bedeutung, und ihr Zusammengehen mit den Uebergangsformen macht die Pappenheim'sche Ansicht plausibel, dass sie in letzteren auch ihre eigenen Entwicklungsformen besitzen.

Aus den engen Wechselbeziehungen der Lymphocylosen und Mononucleosen mit der neutrophilen Leukopenie, sowie dem gewissen Parallelismus der Reaction in gutartigen Leukocytosen ergiebt sich der Werth relativer Bestimmungen dieser Zellklassen im Gesammtleukocytenbilde gerade kliniseh ohne Weiteres.

\section{Schluss.}

An den Schluss unserer Ausführung möchten wir als das hauptsächlichste Ergebniss dieser naturgemäss noch beschränkten Untersuchungen den Satz stellen: nach dem heutigen Stande unserer Kenntnisse kann erst die Leukocytenuntersuchung Anspruch auf wissenschaftliche Vollständigkeit machen, die die drei Factoren,

Gesammtleukocytenzahl,

relative (und eventuell absolute) Zusammensetzung der Zellklassen und

Verschiebung des neutrophilen Blutbildes

in gleicher Weise berücksichtigt.

Dabei darf von dem Grade der Vermehrung oder Verminderung, von der stärkeren oder geringeren Störung des normalen Verhältnisses der Zellarten und endlich von der Höhe der Verschiebung als inconstanten und noch nicht sicher zu beurtheilenden Abstufungen gegenüber dem Gesammttypus des Blutbildes als ungleich wichtiger abgesehen werden.

\section{Literatur.}

1. Grawitz, Klinische Pathologie des Blutes. 1906.

2. Naegeli, Lebrbuch der Blutkrankheiten. 1902-1908.

3. Pappenheim-Ferrata, Fol, haematol. Bd. 10. Archiv 1. 1910.

4. Benjamin, Differentialdiagnose pseudoleukämischer Blutbilder im Kindesalter. Ergebn. d. inneren Med. Bd. 4. 1910.

5. Hess, Blutbefunde bei Milzerkrankungen. Wiener klin. Wochenschr. 1910.

6. Fabian, Werth des Pinkus'schen Zeichens usw. Deutsche med. Wochenschr. 1910.

1) Entgegen der dort hervorgehobenen Angabe habe ich in einigen Fälle echter Auöbenrahr keineswegs Mononucleose finden können. Die Literatur ist schwankend und oft unbrauchbar. 
7. Fabian, Blutbefund der Lymphogranulamatosis. Wiener klin. Wochenschr. 1910.

8. Kocher, Blutuntersuchungen bei Morbus Basedow usw. Langenbeck's Archiv. 1908.

9. Car o, Berliner klin. Wochenschr. 1908.

10. Dunger, Zählung der Eosinophilen und der praktische Werth dieser Untersuchung. Münchener med. Wochenschr. 1910.

11. Stäubli, Die klinische Bedeutung der Eosinophilie. Ergebn. der inneren Med. Bd. 6. 1910.

12. Türck, Klinische Untersuchungen des Blutes bei Infectionskrankheiten. Wien 1910.

13. Grawitz, Ueber myogene Leukocytose. Deutsche med. Wochenschr. 1910.

14. Ziegler u. Schlecht, Leukocytotische Blutveränderungen bei Infectionskrankhoiten. Deutsches Arch. f. klin. Med. Bd. 92. 1908.

15. Bennecke, Die Leukocytose bei Scharlach und anderen Mischinfectionen. Jena 1909 .

16. Arneth, Die neutrophilen weissen Blutkörperchen bei Infectionskrankheiten. Monographie. Jena 1904.

17. Derselbe, Diagnose und Therapie der Anämien. Würzburg 1907.

18. Zoja, Ueber dio Bedentung und den klinischen Werth des Nachweises zwischen Lrythro- und Teukocytolyse usw. Folia haematol. Arch. 10. S. 225. 1910.

19. 'Zelénsli, Ueber das Verhalien des neutrophilen Blutbildes bei gesunden und kranken Säuglingen. Wiener klin. Wochenschr. 1906.

20. Kothe, Das neutrophile Blutbild im Frühstadium der $\Lambda$ ppendicitis. Berliner klin. Wochenschr. 1908.

21. Kothe u. Sonnenburg. Zeitschr. f. Chir. Bd. 100.

22. Kohl, Weitere Mittheilungen über Werth und Bedeutung der Leukocytose und des neutrophilen Blutbildes bei Appendicitis. Bd. 22. 1911.

23. V. Schilling-T'orgau, Ein praktisch und zur Demonstration branchbarer Differentialleukocytometer mit Arneth'scher Verschiebung des Blutbildes. Deutsche med. Wochenschr. 1911. No. 25.

24. Ehrlich-Laıarus, Die Anämien. (Bearb. von Naegeli.) 1909.

25. 'I'ürck-IIelly. Wiener klin. Wochenschr. 1907.

26. Lange, Zur Methodik der Blutuntersuchung bei Puerperalfieber. Zeitschr. f. Geb. u. Gynäkol. Bd. 64.

27. Rosenstern, Exsudative Diathese und Eosinophilie. Jahrb.f. Kinderheik. Bd.69. 1910 .

28. Kownatzki, Blutuntersuchungen bei Puerperalfieber. Beitr. z. Geb. u. Gynäkol. Bd. 10. 1906 .

29. Blumenthal, Frgebnisse der Blutuntersuchungen u.s. w. Beitr. z. Geb. u. Gynäkol. 1907.

30. Albrecht, Die praktische Verwendbarkeit der Leukocytenbestimmung. Zeitschr. f. Geb. u. Gynäkol. Bd. 61. 1907.

31. Gräfenberg, Die prognostische Bedeutung der morphologischen Blutelemente bei pucrperalen Erkrankungen. Arch. f. Gynäk. 1908.

32. Langemeister u. Gans, Der Einfluss der Streptokoklieninfection auf die Leukocytose bei Affen usw. Münchener med. Wochenschr. 1909.

33. Dieselben, Streptoliokkeninfection und Ireukocytose. Monatsschr. f. Geb. u. Gynäliol. Bd. 31. 1910.

34. IIecker, Ueber den Werth relativer Leukocytenzählungen. Münchener med. Wochenschr. 1910.

35. Kothe, Zur Untersuchungsmethode des neutrophilen Biutbildes. Münchener med. Wochenschr. 1909. 
36. Schindler. Zeitschr. f. klin. Med. Bd. 54. 1904.

37. P'ottenger, Phagocytos. in rel. to Arneth' classif. of ncutrophiles. Med. Association. Bd. 52. 1909.

38. Busse, Phagocytose $=$ Arneth'sches Blutbild. Münchener med. Wochenschr. 1910.

39. Arneth, Zum Verständniss des Verhaltens der rothen und weissen Blutkörper bei der Röntgenbehandlung der Leukämie. Münchener mod. Wochenschr. 1905.

40. Arneth, Zum Verständniss der Wirkungen der Röntgenstrahlen bei der Leukämie. Berliner klin. Wochenschr. 1905.

41. Franke, Ueber den Einfluss der Röntgenstrahlen auf den Verlauf der Leukämie. Wiener klin. Wochenschr. 1905.

42. Funk, Zum Verständniss der Besserung der Leukämie durch intercurrente Infectionen. Berliner klin. Wochenschr. 1906.

43. Gothein, Das neutrophile Blutbild bei Malaria. Fol. haenatol. Bd. 11. 1911.

44. Schilling-Torgan, Kritik der Arneth'schen Methodik zur Bestimmung des leukocytären Blatbildes und ihr Werth für Diagnose und Prognose. Fol. haematol. Bd. 12. 1911.

45. Arneth, Die Lungenschwindsucht auf klinischer und experimenteller hänatologischer Grundlage. Monographie u. Zeitschr. f. Tuberc. u. Heilstättenw. Bd. 7. H. 4 u. 5 .

46. Röver, Ueber 25 mit Marmorek's Serum behandelte Fälle von T'uberculose. Beitr. z. Klinik d. Tuberc. Bd. 6. 1906.

47. Uhl, Ueber dio neutrophile Leukocytose bei specifischer Therapie der chronischen Lungentaberculose. Beitr. z. Klinik der Tubero. 1906.

48. Dluski und Rospedzikowski, Blutuntersuchungen nach der Arneth'schen Methode im Allgemeinen und bei der Tuberculose im Speciellen. Beitr. z. Klin. der T'uberc. Bd. 14.

49. v. Bonsdorff, Ueber das Arneth'sche neutrophile Blutbild und de Bedeutung für die Lungentuberculose. Fol. haematol. Bd. 9. 1910.

50. Busse, Die Leukocytose. Habilitationsschrift. Berlin 1908.

51. Sondern, Werth der Blutuntersuchungen in der chirurgischen Diagnose. 'Zeitschrift f. Chir. Bd. 102. 1910.

52. Baradulin, Ueber Blutveränderungen bei malignen Neubildungen. Fol. haematol. Bd. 9. 1910.

53. Rieux, De grand mononucléaire du sang et de ses variations dans les divers états pathologiques. Fol. haematol. Bd. 10. 1910.

54. Patolla, Uober die endotheliale Natur der grossen Mononucleären des Blutes. Deutsche med. Wochenschr. 1910. Monographie. Siena. 1908.

\section{Erklärung der Abbildungen auf Tafel XXIV-XXVI.}

Die Abbildungen sind photographische Aufnahmen nach meinem Differentialleulsocytometer in etwa $1 / 5$ natürlicher Grösse der Mehrzahl nach. Sie sind im allgemeinen nach den schriftlich gewonnenen Durchschnittsbildern aus 500 Zellen eingestellt, um eine grössere Gleichmässigkeit zu gewährleisten. Die nähere Besprechung findet sich auf den bezeichneten Seiten.

Tafel XXIV, $\Lambda \mathrm{bb} .1-4$.

Abbildung 1: Gesammtansicht des Differentialleukocytometers (etwa $1 / 10$ natürlicher Grösse) mit bunter Zelltafel und eingestelltem Normalblutbild. (S. 692.)

Abbildung 2: Farbige Zolltafel (etwa 1/2 natürlicher Grösse). Die wichtigen charakteristischen Zollformen der Leukocyten in mehreren Typen mit Bezeichnung. (S. 693.) 
Abbildung 3: Die "Normale" des Apparates eingestellt. Physiologische Schwankungszahlen der relativen $\mathrm{pCt}$. Werte. (Am Apparat, grün, daher hier etwas blass.) Scaleneinteilung. (S. 695.)

Abbildung 4: Degl. Einstellung einer Eosinophilie mit geringer Monucleose bei normaler Leukocytenzahl (6000). (Chronische Ankylostomiasis.) (S.696.)

Tafel XXIV, $\Lambda \mathrm{bb} .5 \mathrm{a}--\mathrm{k}$.

Fall von leichter Appendicitis mit späterer Complication durch Peritonitis. (S. 696,700 u. 708.)

Abbildung 5a: $\mathrm{I}=10000$. Neutrophilie mit geringer Verschiebung. (Vor Operation.)

Abbildung 5b: $L=7500$. Ausgeprägte Neutrophilie ohne Verschiebung. Aneosinophilie (relativ). I.ymphopenie.

Abbildung 5c: L = 9400. Neutrophilie mit Versehiebung nach "rechts". Relative Lymphopenie. Leichte Mononucleose. Aneosinophilie (relativ).

Abbildung 5d: $\mathrm{L}=13000$. Zunehmende Gesammtleukocytose mit weiterer Besserung des Blutbildes. Neutrophilie mit Verschiebung nach "rechts". Lymphocyten absolut hochnormal. thosinophile fast normal.

Abbildung 5e: $\mathrm{L}=9500$. Abnchmende Gesammtleakocytose. Sinkende Neutrophilie. Leichte Mon on ucleose. Eosinophile und Jymphocyten fast normal. Annäherung an die Normale.

Abbildung 5f: L $=11700$. Anstieg der Gesanmtleukocytose mit deutlicher regenerativer Verschiebung. Eosinophile und Lymphocyten gut vertreten, Reizung mit günstiger Prognose (Wundabscess). Der 7. Krankheitstag fehlt.

Abbildung 5g: 8. Krankheitstag. $\mathrm{L}=12400$.

Abbildung 5h: 17. Krankheitstag. $\mathrm{I}_{4}=13400$. Fortbestehen der leichten Reizung mit regenerativ wenig verschobenem Blutbild; Eosinophile und Jymphocyten nachgeholt trotz Wundeiterung. Desgl. bis zum 20. Krankheitstage.

Abbildung 5i: 22. Krankheitstag. $\mathrm{L}=33800$. Hochschnellende Gesammthyperleukocytose. Neutrophilie mit schwer regenerativer Verschiebung. Lymphopenie.Anesinophilie (fast absolut). (Peritonitis-Complication.)

Abbildung 5k: 23. Krankheitstag. $L==25800$. Sinkende Gesammtzahl mit weitererschwer-regenerativer und degeneratiververschiebung. Relative Lymphopenie und fehlende Mononucleäre. AbsoluteAn. eosinophilie. (Abends: 'lod.)

Tafel XXV, Abb. 1a-i.

Schwere Sepsis durch Scheuerwunde am Knöchel. Fortlaulend täglich be. obachtet. (S. 697, 701 u. 708.)

Abbildunga: $\mathrm{L}=11000$. Hyperleukocytose. Starke regenerative Verschiebung bei geringer Neutrophilie. Lymphocyten absolut fast normal. Aneosinophilie (relativ).

Abbildung $b: L=15000$. If yperleukocytose vermehrt bei gleichem Blutbilde. Etwas gebessert.

Abbildung c: $L=12000$. Hyperleukocytose vermindert bei Zunahme der Verschiebung. $\Lambda$ bsolute Lymphopenie. Aneosinophilie (fast absolut). Prognose sehr ernst.

Abbildung d: $\mathrm{L}=7100$. Hyperleukocytoso stark im Absinken. Verschiebung schwer degenerativ and regenerativ. $\Lambda$ neosin ophilie absolut. Lymphopenie verstärkt. Anisonormocytose Arneth's. Prognose infaust. (Amputatio femoris). 
$\Lambda$ bbildung e: $I_{A}=5100$. I eukopenie mit schwerer septisch-regenerativer Verschiebung. Absalute Aneosinophilie. Lymphocyten etwas gebessert; leichte Mononucleose (nicht relativ). Anisohypoleukocytose Armeth's.

Abbildung $\mathrm{f}: \mathrm{L}=14000$. Leukämoides Blutbild dureh Insufficienz des Knochenmarkes. Schwerste Verschiebung. Absolute Aneosinophilie. Schwere Lymphopenie. Abends: 'T'od.

Tafel XXV, Abb. 2-9.

Abbildung 2: Myeloische Leukämie. $L_{s}=100000$. Breite durchaus regenerative Verschicbung bei starker Neutrophilia. Eosinophile entsprechend vermehrt. Relative Lymphopenie. Mononucleäre entsprechend vermehrt. (S.696,703.)

Abbildung 3: Myeloische Leukämie. $L=60000$. Gleicher Befund. Mononucleose (theilweise anscheinend durch Promyelocyten oder $\Lambda$ granulocyten). (S. 703.)

A bbildung 4 und 5: Malaria-Tertiana-Recidiv. 1. und 2. Tag. (S. 704, 709.) 4. $\mathrm{L}=6300$. Deutliche de- und regenerative Verschiebung. Hypeosinophilie. Mononueleose. In Anlall bei zahlreichen P'arasiten. Chininkur begonnen. 5. $\mathrm{J}=4300$. leakopenic. Sehr starlie Lymph. ocytose. Mässige degenerative Verschiebung bei absoluter Verminderung der Neutrophilen. Mononucleose. Hypeosinophilie. Noch Theilungen. Fieber fast beseitigt. Chinin-Effect.

Abbildung 6: Chronische Malaria: Tertiana. 1. Tag nach dem Anfall unter Chinin. (S. 704, 709.) $\mathrm{l}=3400$. Leukopenie. Sehr starke Mononucleose und relative Lymphocytose. Starke degenerative Verschiebung der verminderten Neutrophilen. Ilypeosinophilie.

Abbildung 7: Desgl. aber bereits ohne Parasiten. $L=\$ 900$. Leukopenie. Sehr starke Mononucleose. Relative Lymphocylose. Noch etwas degenerative deutliche Verschiebung. Relative Aneosinophilie. (S. 704, 709.)

Abbildung 8: Malaria: Tropica frisoh. Gut inficitt. 1. u. 2. Tag hoobnormale Leukocytose. $(7100,6700$.$) \quad (S. 704, 709.) 3. 'l'ag nach dem$ $\Lambda \mathrm{nfall}$ : Chinin seit Anfall; Parasiten beseitigt. $\mathrm{I}=3800$. Also etwas verspäleter $\Lambda$ bsturz zur leukopenie. Geringe Verschicbung. Sehr starke Mononucleose und relative Lymphoeytose. Ancosinophilie (relativ).

Abbildung 9: Malaria: Chronische Tertiana-Remission. (\$.705.) 12. Tag nach Anfall. Erythrocyten von 1675000 gestiegen auf fast 3000000 . Leukocyten von 3300 gestiegen auf 6200 . Lymphocy tose. Abgesunkene Mononucleose. Eosinophile und Basophile stets vorhanden, zeitweise etwas vermehrt. Verschiebung "nach rechts".

\section{Tafel XXVI.}

Abbildung 1: Leberabscess-Verdacht. (S. 706). $\mathrm{h}=5900 . \quad$ Ohne Verschiebung auch relativ fast normales Blutbild. Starke degenerative (chronische) Verschiebung. Prognose abwartend.

$\Lambda$ bbildung 2: Typhus abdominalis. 3. Woche. Nachschub. (S.710.) $\mathrm{T}_{1}=4000$. Leukopenie. Starke rein degenerative Verschiebung. Absolute Lymphocytase. Relative Mononucleose. Fast absolute Aneosinophilie.

Abbildung 3: Typhus abdominalis: 3 . Woche; mittelschwer. $\mathrm{L}=3000$. Leukopenie. Lymphocytose hier relativ. Verschiebung sehr stark, degenerativ. Fast absolute Aneosinophilie. Relative Mononucleose, (S. $706,708$. 
Leukocytenblutbilder mit Einbeziehung d. vereinfachten Arneth'schen Methode usw. 715

Abbildung 4: Beri-Beri. Tod an Herzschlag 2 Tage darauf. (S. 709.) $\mathrm{L}=$ 5400. Fast fehlende Verschiebung. Absolute Lymphocytose. Absolute Mononucleose.

Abbildung 5: Schwere Verbrennung mit Wunderysipel in Abheilung. (S. 707.) $L=25000$. Starke Hyperleukocytose. Neutrophilie mit sehr geringer Verschiebung. Relative Aneosinophilie. Gut folgende Lymphocytose. Prognose für Sepsis sehr günstig.

A bbildung 6: Schwere Syphilis mit Nephritis, Cylindrurie; drohende Anurie. $\mathrm{L}=10600$. Hyperleukncytose. (S. 706.) Ausgesprochene Neutrophilie mit geringer Verschiebung und absoluter Lymphopenie. Fast absolute Aneosinophilie. Schlechte Uebereinstimmung des neutrophilen Blutbildes, bessere der Nebenklassen mit dem Gesammtzustand. Diagnostisch z. B. Sepsis, eitrige Nephritis auszuschliessen.

Abbildung 7: Mittelschwere Pneumonie. (S. 706). IL $=24800$. Neutrophilie mit breiter regenerativer Verschiebung. Relative Lymphopenie und Aneosinophilie. Mononucleose gut entsprechend. Prognose abwartend, mit Ansteigen der Lymphocyten und Eosinophilen gut. 
Zeitschrift f. exp. Path. u. Therapie, IX. Bd.
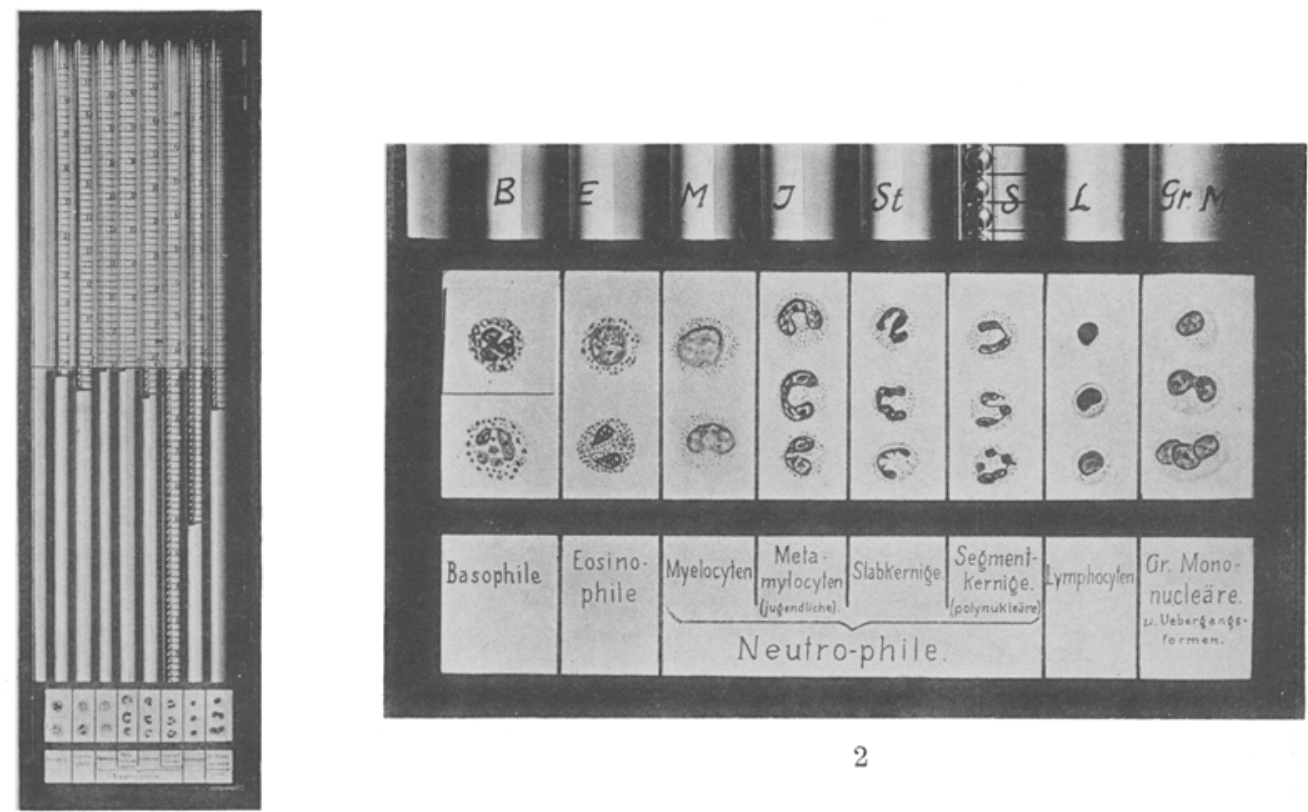

2

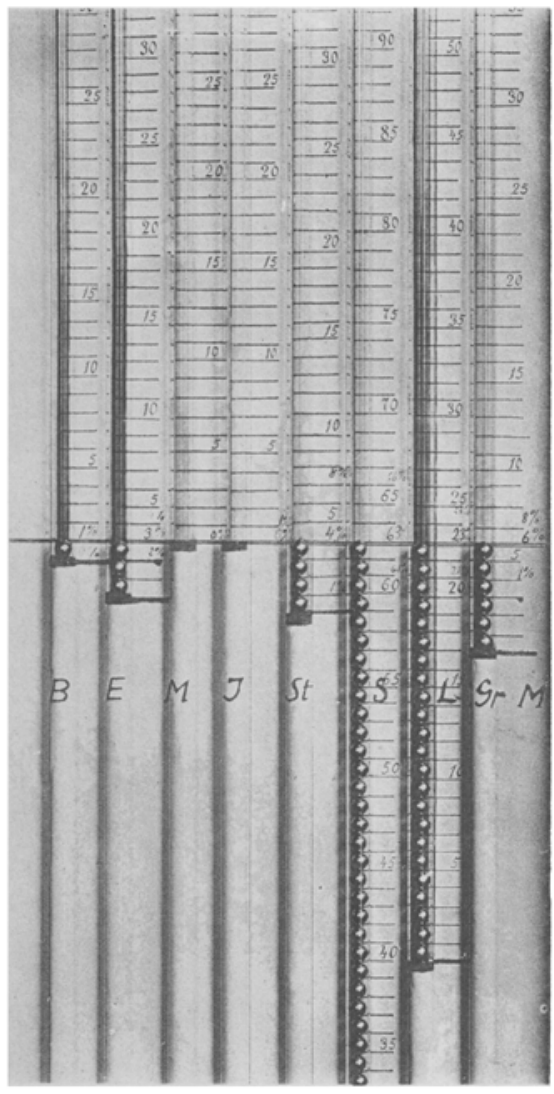

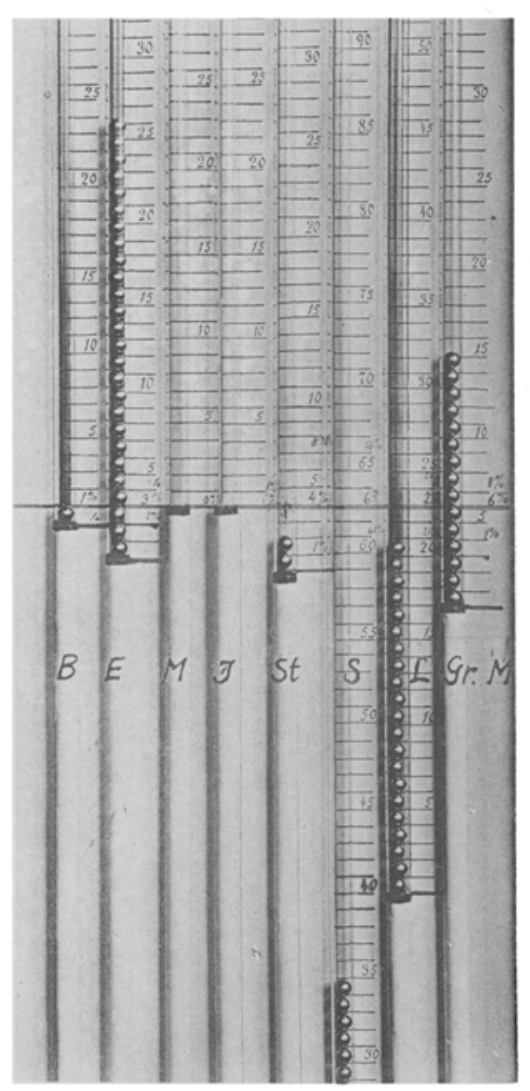

4 

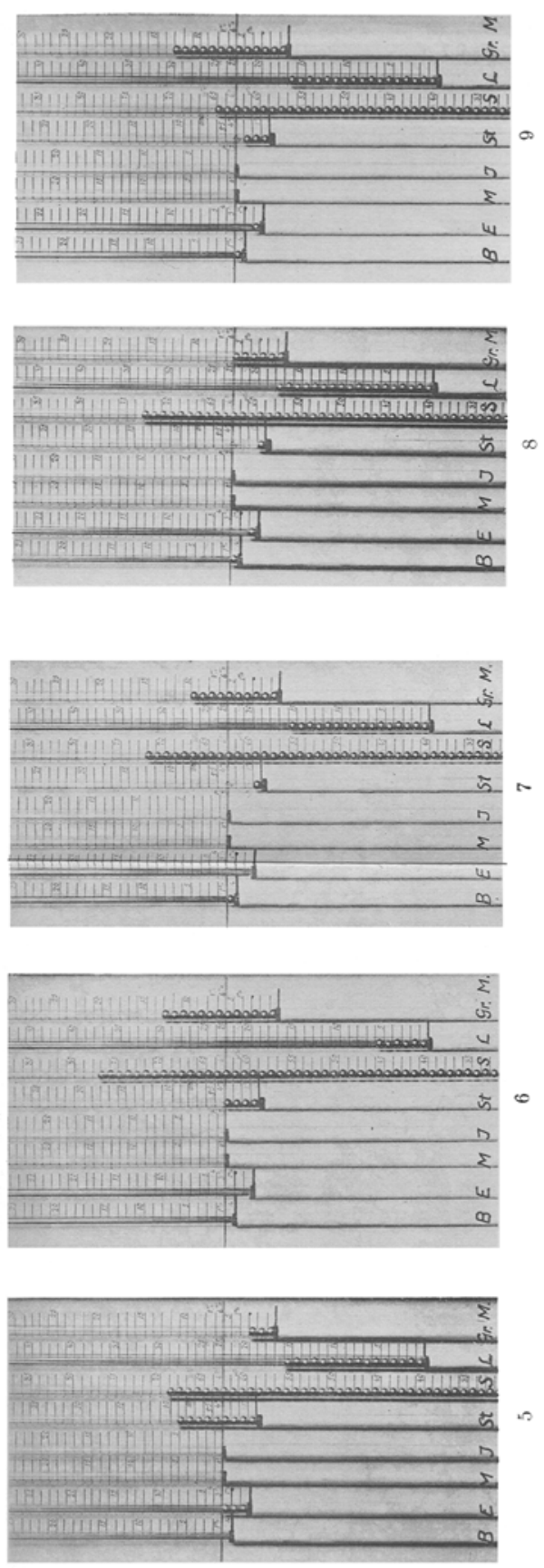
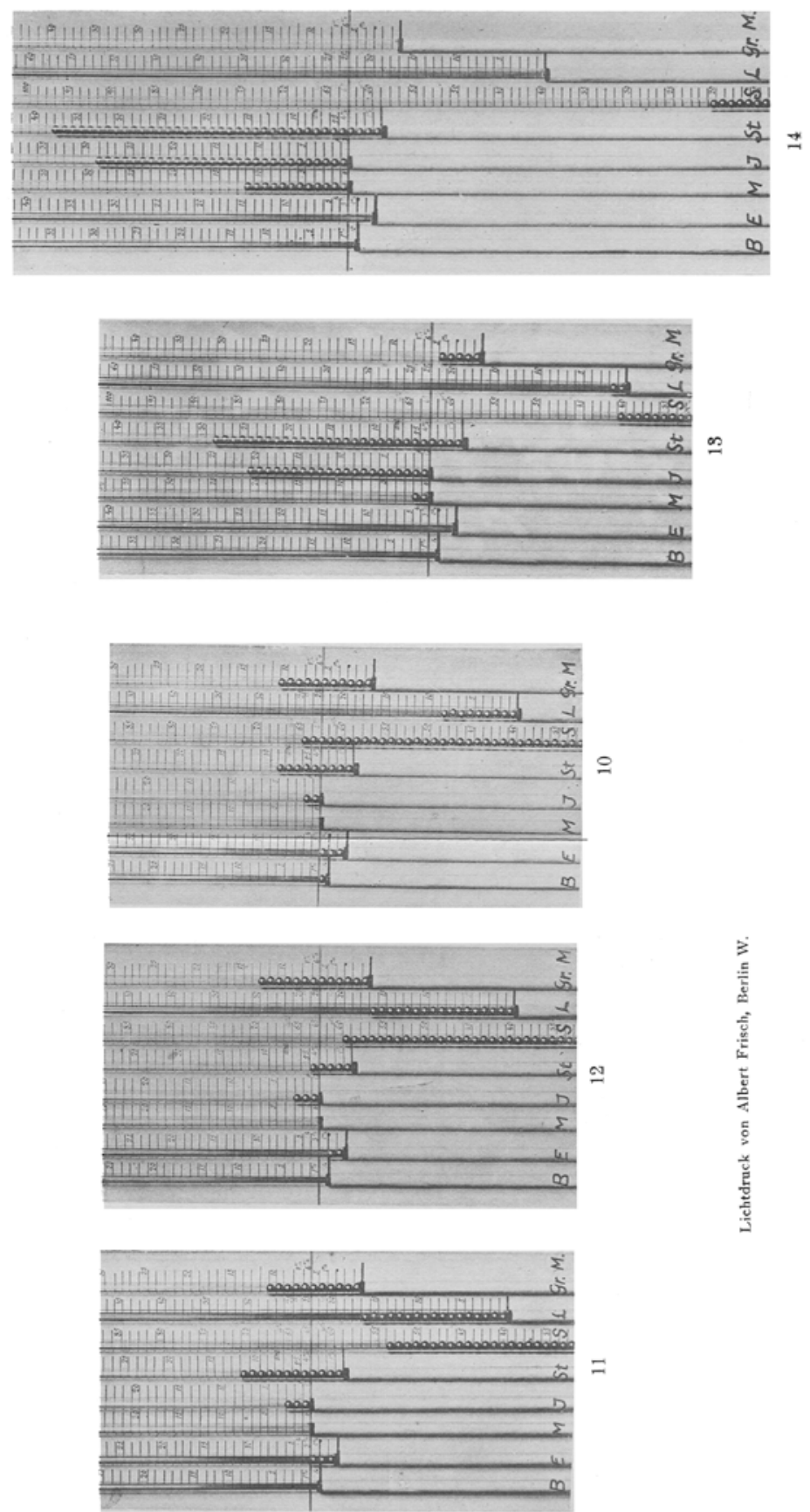


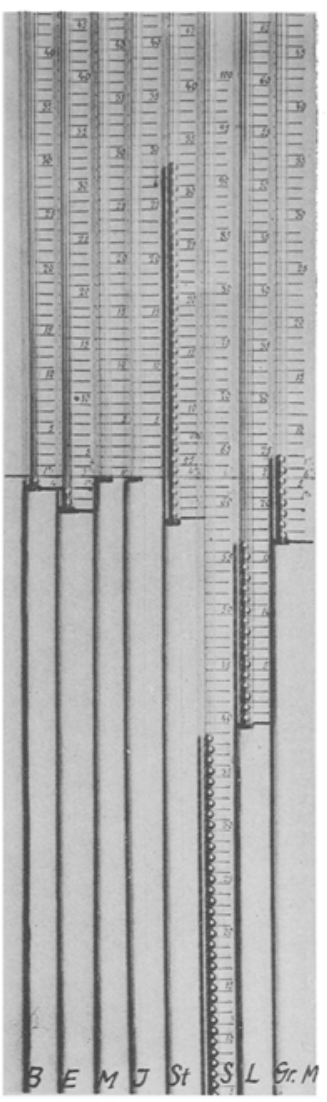

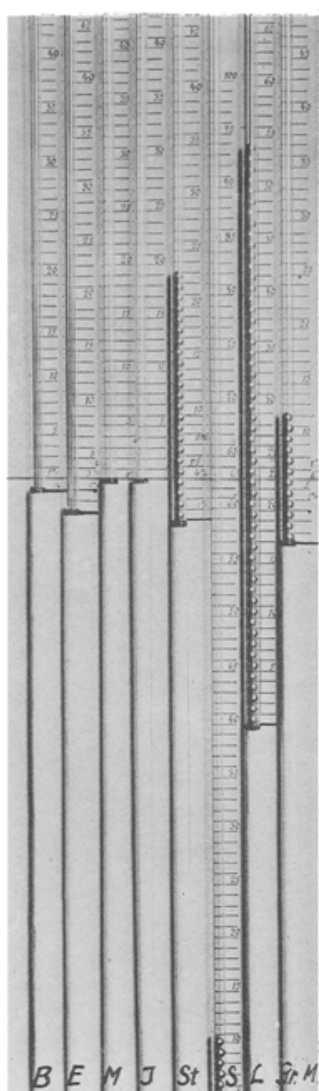

2

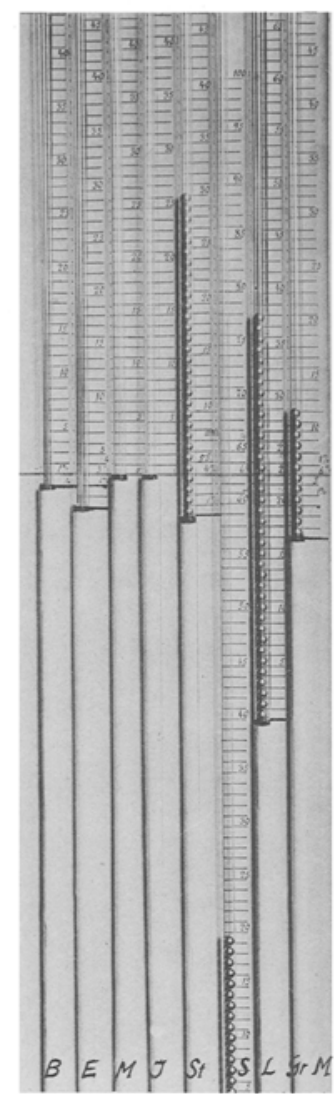

3

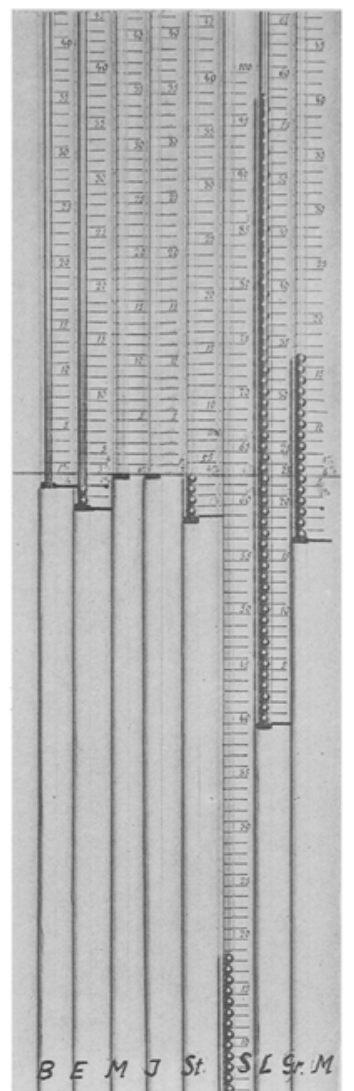

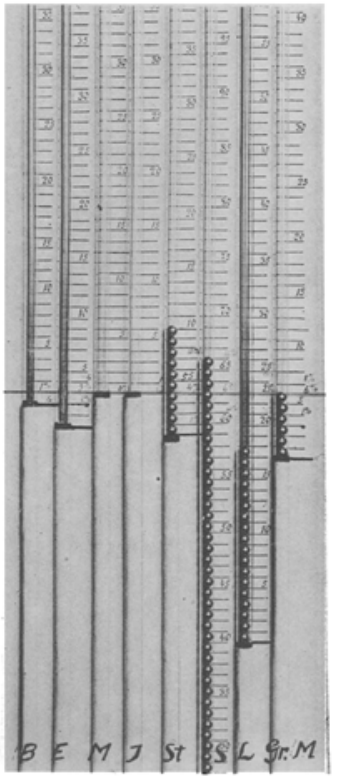

5

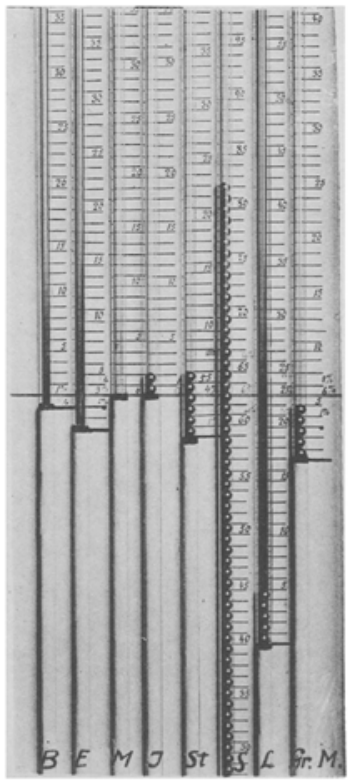

6

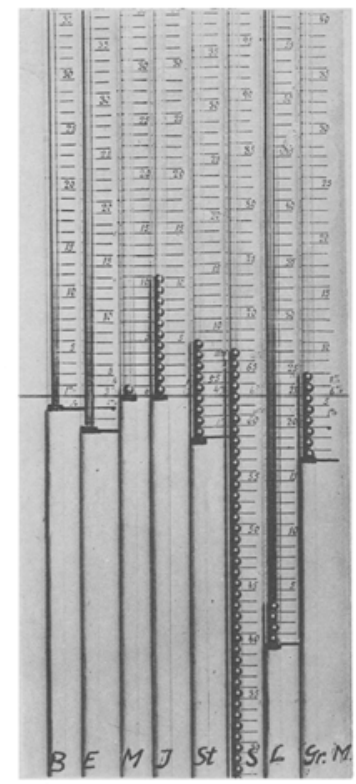

Lichtdruck von Albert Frisch, Berlin W. 